JOURNAL OF THE

AMERICAN MATHEMATICAL SOCIETY

Volume 20, Number 3, July 2007, Pages 675-699

S 0894-0347(06)00552-2

Article electronically published on November 17,2006

\title{
CONVERGENCE OF KÄHLER-RICCI FLOW
}

\author{
GANG TIAN AND XIAOHUA ZHU
}

\section{INTRODUCTION}

In this paper, we prove a theorem on convergence of Kähler-Ricci flow on a compact Kähler manifold $M$ which admits a Kähler-Ricci soliton. A Kähler metric $h$ is called a Kähler-Ricci soliton if its Kähler form $\omega_{h}$ satisfies equation

$$
\operatorname{Ric}\left(\omega_{h}\right)-\omega_{h}=L_{X} \omega_{h},
$$

where $\operatorname{Ric}\left(\omega_{h}\right)$ is the Ricci form of $h$ and $L_{X} \omega_{h}$ denotes the Lie derivative of $\omega_{h}$ along a holomorphic vector field $X$ on $M$. As usual, we denote a Kähler-Ricci soliton by a pair $\left(g_{K S}, X\right)$. According to [TZ1, $X$ should lie in the center of a reductive Lie subalgebra $\eta_{r}(M)$ of $\eta(M)$, which consists of all holomorphic vector fields on $M$. If $X=0, \omega_{h}$ is just a Kähler-Einstein metric. Since $\omega_{h}$ is $d$-closed, we may write $L_{X} \omega_{h}=\frac{\sqrt{-1}}{2 \pi} \partial \bar{\partial} \theta$ for some real-valued smooth function $\theta$. It follows that the first Chern class $c_{1}(M)$ is positive and it is represented by $\omega_{h}$.

The Ricci flow was first introduced by R. Hamilton in $\mathrm{Ha}$. If the underlying manifold $M$ is Kähler with positive first Chern class, it is more natural to study the following Kähler-Ricci flow (normalized):

$$
\left\{\begin{aligned}
\frac{\partial g(t, \cdot)}{\partial t} & =-\operatorname{Ric}(g(t, \cdot))+g(t, \cdot), \\
g(0, \cdot) & =g_{0},
\end{aligned}\right.
$$

where $g_{0}$ is a given metric with its Kähler class representing $c_{1}(M)$. It can be shown that (0.1) preserves the Kähler class.

Let $\operatorname{Aut}_{r}(M)$ be the connected Lie subgroup of the automorphism group of $M$ corresponding to $\eta_{r}(M)$. Let $K$ be a maximal compact subgroup of $\operatorname{Aut}_{r}(M)$. According to [TZ1], we may assume that a Kähler-Ricci soliton $\left(g_{K S}, X\right)$ is $K$ invariant and the imaginary part $\operatorname{Im}(X)$ of $X$ generates a one-parameter subgroup $K_{X}$ of $K$. The following is our main result.

Main Theorem. Let $M$ be a compact Kähler manifold which admits a KählerRicci soliton $\left(g_{K S}, X\right)$. Then any solution $g(t, \cdot)$ of $(0.1)$ will converge to the $g_{K S}$ in the sense of Cheeger-Gromov if the initial Kähler metric $g_{0}$ is $K_{X}$-invariant.

Corollary. If $M$ admits a Kähler-Einstein metric, then any solution of (0.1) will converge to a Kähler-Einstein metric in the sense of Cheeger-Gromov for any initial Kähler metric $g_{0}$ with Kähler class $c_{1}(M)$.

Received by the editors August 29, 2005.

2000 Mathematics Subject Classification. Primary 53C25; Secondary 32J15, 53C55, 58E11.

The first author was partially supported by an NSF grant and a Simon fund.

The second author was partially supported by NSF grant 10425102 in China and a Huo Y-D fund.

(C)2006 American Mathematical Society Reverts to public domain 28 years from publication 
This corollary was first announced by G. Perelman [P2] when he was visiting MIT in the Spring of 2003.

Our proof of the main theorem is to study a certain complex Monge-Ampère flow which arises from (0.1). The flow of this type has been studied before by many people (cf. Ca, CT1, CT2]). Indeed, our proof used a deep estimate of Perelman ([P2]; also see [ST]). We combined Perelman's estimate with estimates on solutions of complex Monge-Ampère equations we have used in our proving the uniqueness of Kähler-Ricci solitons TZ1.

The organization of this paper is as follows. In Section 1, we describe an unpublished estimate of Perelman on the time derivative of potential functions of evolved Kähler metrics along the Kähler-Ricci flow. In Section 2, we use the relative capacity theory, which was first developed in $\mathrm{Ko}$ and adapted to the case of Kähler-Ricci solitons in [TZ1, to obtain a $C^{0}$-estimate for a certain Monge-Ampère equation. Then in Section 3, we obtain a global Harnack-type inequality for solutions of complex Monge-Ampère flow associated to the Kähler-Ricci flow (0.1). In Section 4, we derive monotonicity of the generalized K-energy introduced in TZ2]. A $C^{0}$-estimate on a modified complex Monge-Ampère flow is obtained in Section 5 and the Main Theorem will be proved in Section 6 .

\section{An estimate of Perelman}

In this section, we first reduce the Kähler-Ricci flow to a fully nonlinear flow on Käher potentials. Then we discuss a recent and deep estimate of Perelman.

Let $(M, g)$ be an $n$-dimensional compact Kähler manifold with its Kähler form $\omega_{g}$ representing the first Chern class $c_{1}(M)>0$. In local coordinates $z_{1}, \cdots, z_{n}$, we have

$$
\omega_{g}=\frac{\sqrt{-1}}{2 \pi} \sum_{i, j=1}^{n} g_{i \bar{j}} d z^{i} \wedge d \bar{z}^{j}, g_{i \bar{j}}=g\left(\frac{\partial}{\partial z_{i}}, \frac{\partial}{\partial z_{j}}\right) .
$$

Moreover, the Ricci form $\operatorname{Ric}\left(\omega_{g}\right)$ is given by

$$
\left\{\begin{array}{l}
R_{i \bar{j}}=-\partial_{i} \bar{\partial}_{j} \log \left(\operatorname{det}\left(g_{k \bar{l}}\right)\right), \\
\operatorname{Ric}\left(\omega_{g}\right)=\frac{\sqrt{-1}}{2 \pi} \sum_{i, j=1}^{n} R_{i \bar{j}} d z^{i} \wedge d \bar{z}^{j} .
\end{array}\right.
$$

Since the Ricci form represents $c_{1}(M)$, there exists a smooth function $h$ on $M$ such that

$$
\operatorname{Ric}\left(\omega_{g}\right)-\omega_{g}=\frac{\sqrt{-1}}{2 \pi} \partial \bar{\partial} h .
$$

An easy computation shows that the flow (0.1) preserves the Kähler class of its solution $g(t)$, so we may write the Kähler form of $g(t)$ at a solvable time $t$ as

$$
\omega_{\varphi}=\omega_{g_{0}}+\frac{\sqrt{-1}}{2 \pi} \partial \bar{\partial} \varphi
$$

for some smooth function $\varphi=\varphi(t, \cdot)=\varphi_{t}$. This $\varphi$ is usually called a Kähler potential function associated to the Kähler metric $g(t)$. Using the Maximal Principle, one can show that (0.1) is equivalent to the following complex Monge-Ampère flow 
for $\varphi(t, \cdot)$ :

$$
\left\{\begin{array}{l}
\frac{\partial \varphi}{\partial t}=\log \frac{\operatorname{det}\left(g_{i \bar{j}}+\varphi_{i \bar{j}}\right)}{\operatorname{det}\left(g_{i \bar{\jmath}}\right)}+\varphi-h, \\
\varphi(0, \cdot)=0
\end{array}\right.
$$

Observe that $\left.\frac{\partial \varphi}{\partial t}\right|_{t=0}=-h$.

Differentiating on both sides of (1.2) on $t$, we have

$$
\frac{\partial}{\partial t} \frac{\partial \varphi}{\partial t}=\Delta^{\prime} \frac{\partial \varphi}{\partial t}+\frac{\partial \varphi}{\partial t}
$$

where $\Delta^{\prime}$ denotes the Laplacian operator associated to the metric $\omega_{\varphi}$. Then it follows from the standard Maximal Principle that

$$
\left|\frac{\partial}{\partial t} \varphi(t, \cdot)\right| \leq C e^{t}
$$

and consequently,

$$
|\varphi(t, \cdot)| \leq C e^{t} .
$$

By using these facts and arguments in deriving the higher order estimates in Yau's solution of the Calabi conjecture [Ya], H.D. Cao showed that (1.2) is solvable for all $t \in(0,+\infty)$ Ca].

Using his $W$-functional and arguments in proving noncollapsing of the Ricci flow [P1], recently, G. Perelman proved the following deep estimate [P2].

Lemma 1.1. Let $\varphi_{t}$ be a solution of Monge-Ampère flow (1.2). Choose $c_{t}$ by the condition $h_{t}=-\frac{\partial \varphi}{\partial t}+c_{t}$ such that

$$
\int_{M} e^{h_{t}} \omega_{\varphi_{t}}^{n}=\int_{M} \omega_{g_{0}}^{n} .
$$

Then there is a uniform constant $A$ independent of $t$ such that

$$
\left|h_{t}\right| \leq A .
$$

For the reader's convenience, we will present a proof of Lemma 1.1 taken from ST in the appendix. Lemma 1.1 is crucial in proving our main theorem. Recall that $h_{t}$ is defined by (1.1) with $\omega_{g}$ replaced by $\omega_{\varphi_{t}}$ and can be different from a constant. In Section 4 below we will further prove that $c_{t}$ is uniformly bounded and so $\frac{\partial \varphi}{\partial t}$ is.

\section{Relative Capacity and $C^{0}$-estimate}

In this section, as in TZ1, we will use the relative capacity theory for plurisubharmonic functions first developed in $\mathrm{Ko}$ to derive a $C^{0}$-estimate on a certain Monge-Ampère equation.

First we recall some notation which can be found in $\overline{B T}$. For any compact subset $K$ of a strictly pseudoconvex domain $\Omega$ in $\mathbb{C}^{n}$, its relative capacity in $\Omega$ is defined as

$$
\operatorname{cap}(K, \Omega)=\sup \left\{\int_{K}(\sqrt{-1} \partial \bar{\partial} u)^{n} \mid u \in \operatorname{PSH}(\Omega),-1 \leq u<0\right\},
$$

where $\operatorname{PHS}(\Omega)$ denotes the space of plurisubharmonic functions (abbreviated as psh) in the weak sense. For any open set $U \subset \Omega$, we have

$$
\operatorname{cap}(U, \Omega)=\sup \{\operatorname{cap}(K, \Omega) \mid \text { for any compact } K \subset U\} .
$$


The extremal function of $K$ relative to $\Omega$ is defined by

$$
u_{K}(z)=\sup \left\{u(z) \mid u \in \operatorname{PSH}(\Omega) \cap L^{\infty}(\Omega), u<0 \quad \text { and }\left.\quad u\right|_{K} \leq-1\right\} .
$$

One can show that $u_{K}^{\star}(z)=\varlimsup_{z_{z^{\prime} \rightarrow z}} u_{K}\left(z^{\prime}\right)$ is a psh function. It is called the upper semicontinuous regularization of $u_{K}$. A compact set $K$ is said to be regular if $u_{K}^{\star}=u_{K}$. The following are some properties of $u_{K}^{\star}$ (cf. [BT], [AT]):

$$
\begin{aligned}
& u_{K}^{\star} \in \operatorname{PSH}(\Omega),-1 \leq u_{K}^{\star} \leq 0, \lim _{z \rightarrow \partial \Omega} u_{K}^{\star}=0, \\
& \left(\sqrt{-1} \partial \bar{\partial} u^{\star}\right)^{n}=0 \text { on } \Omega \backslash K, \\
& u_{K}^{\star}=-1 \text { on } \mathrm{K} \text {, except on a set of relative capacity zero. }
\end{aligned}
$$

Moreover, we have

$$
\operatorname{cap}(K, \Omega)=\int_{\Omega}\left(\sqrt{-1} \partial \bar{\partial} u_{K}^{\star}\right)^{n}=\int_{K}\left(\sqrt{-1} \partial \bar{\partial} u_{K}^{\star}\right)^{n} .
$$

Lemma 2.1. Let $\Omega$ be a strictly pseudoconvex domain in $C^{n}$ and let $u<0$ be a smooth solution of the following complex Monge-Ampère equation on $\Omega$ :

$$
\operatorname{det}\left(u_{i \bar{j}}\right)=f
$$

Suppose that $u$ and $f$ satisfy

$$
\begin{aligned}
& u(p)>c(p \in \Omega) \quad \text { and } \\
& \int_{K} f d v \leq \operatorname{Acap}(K, \Omega) \frac{\operatorname{cap}(K, \Omega)^{\frac{1}{\delta}}}{1+\operatorname{cap}(K, \Omega)^{\frac{1}{\delta}}}
\end{aligned}
$$

for any compact subset $K$ of $\Omega$. If the sets

$$
U(s)=\{z \mid u(z)<s\} \cap \Omega^{\prime \prime}
$$

are nonempty and relatively compact in $\Omega^{\prime \prime} \subset \Omega^{\prime} \subset \subset \Omega$ for any $s \in[S, S+D]$, where $S$ is some number, then there is a uniform constant $C$, which depends only on $c, D, \delta, \Omega^{\prime}, \Omega$, such that

$$
-\inf _{\Omega^{\prime \prime}} u \leq C A^{\delta}+D
$$

Proof. This lemma is essentially due to $[\mathrm{Ko}$. For the reader's convenience, we will include a proof using an argument from [TZ1]. Put

$$
a(s)=\operatorname{cap}(U(s), \Omega) \quad \text { and } \quad b(s)=\int_{U(s)}(\sqrt{-1} \partial \bar{\partial} u)^{n} .
$$

Then we define an increasing sequence $s_{0}, s_{1}, \ldots, s_{N}$ by setting $s_{0}=S$ and

$$
s_{j}=\sup \left\{s \mid a(s) \leq \lim _{t \rightarrow s_{j-1}^{+}} e a(t)\right\}
$$

for $j=1, \ldots, N$, where $N$ is chosen to be the greatest integer such that $s_{N} \leq S+D$. By using an argument in Lemma 4.1 of [TZ1], we can prove

$$
S+D-s_{N} \leq(A e)^{\frac{1}{n}} a(S+D)^{\frac{1}{n \delta}}
$$

and

$$
s_{N}-S \leq 2(A e)^{\frac{1}{n}}(1+n \delta) a(S+D)^{\frac{1}{n \delta}} .
$$

However, it was proved in [AT] (or Theorem 1.2.11 in [Ko] that

$$
\operatorname{cap}\left(\{u<s\} \cap \Omega^{\prime}, \Omega\right) \leq \frac{c^{\prime}}{|s|},
$$


where $c^{\prime}$ depends only on $c$ and $\Omega^{\prime}$. It implies that

$$
a(S+D) \leq \frac{c^{\prime}}{-D-S} .
$$

Combining (2.4)-(2.6), we get

$$
D \leq 2(2+n \delta)(A e)^{\frac{1}{n}}\left(\frac{c^{\prime}}{-D-S}\right)^{\frac{1}{n \delta}} .
$$

It follows that

$$
-S \leq c^{\prime}\left(\frac{2(2+n \delta)}{D}\right)^{n \delta} e^{\delta} A^{\delta}+D .
$$

Consequently, we have

$$
-\inf _{\Omega^{\prime \prime}} u \leq c^{\prime}\left(\frac{2(1+n \delta)}{D}\right)^{n \delta} e^{\delta} A^{\delta}+D,
$$

so $(2.3)$ is proved.

Lemma 2.2. Let $\Omega$ be a strictly pseudoconvex domain in $C^{n}$ and let $u<0$ be a smooth solution of the following complex Monge-Ampère equation on $\Omega$ :

$$
\operatorname{det}\left(u_{i \bar{j}}\right)=f
$$

where $f(\geq 0) \in L^{1+\epsilon_{0}}(\Omega)$ for some $\epsilon_{0}>0$. Suppose that $u$ satisfies

$$
u(p)>c(p \in \Omega) .
$$

Define $U(s)$ as in last lemma. If the $U(s)$ are nonempty and relatively compact in $\Omega^{\prime \prime}$ for any $s \in[S, S+D]$ for some $S$, then for any positive $\delta \leq \delta_{0}$ and $\epsilon \leq \epsilon_{0}$, there is a uniform constant $C=C\left(c, D, \delta_{0}, \epsilon_{0}, \Omega^{\prime}, \Omega\right)$ such that

$$
-\inf _{\Omega^{\prime \prime}} u \leq C\left(\frac{1}{\delta \epsilon}\right)^{n+\delta}\|f\|_{L^{1+\epsilon}(\Omega)}^{\delta}+D .
$$

Proof. Let $u_{K}$ be the relative extremal function of a regular set $K$ with respect to $\Omega$ and let $v=\operatorname{cap}^{-\frac{1}{n}}(K, \Omega) u_{K}$. Then $v$ is a psh function and satisfies

$$
\int_{\Omega}(\sqrt{-1} \partial \bar{\partial} v)^{n}=1 \quad \text { and } \quad \lim _{z \rightarrow \partial \Omega} v=0
$$

By Lemma 2.5.1 in [Ko], we have

$$
\lambda\left(U^{\prime}(s)\right) \leq c^{\prime} \exp \{-2 \pi|s|\}
$$

for some uniform constant $c^{\prime}$ independent of $v$, where $\lambda\left(U^{\prime}(s)\right)$ is the Lebseque measure of $U^{\prime}(s)=\{v<s\}$. It follows that for any $q \geq 1$,

$$
\begin{aligned}
\int_{\Omega}|v|^{q} d \mu & \leq|\Omega|+\sum_{i=1}^{\infty} \int_{-s-1 \leq v \leq-s}|v|^{q} d \mu \\
& \leq|\Omega|+c^{\prime} \sum_{i=1}^{\infty}(s+1)^{q} e^{-2 \pi s} \\
& \leq|\Omega|+c^{\prime} e^{4 \pi} \int_{2}^{+\infty} s^{q} e^{-2 \pi s} d s \\
& \leq C_{1} 2^{q+2}([q]+2) ! \leq C_{1} 2^{q+2}(q+2)^{q+2} .
\end{aligned}
$$


On the other hand, we have

$$
\begin{aligned}
& \operatorname{cap}(K, \Omega)^{-1}\left(1+\operatorname{cap}^{-1 / \delta}(K, \Omega)\right) \int_{K} f d \mu \\
& \leq \int_{K}|v|^{n}\left(1+|v|^{\frac{n}{\delta}}\right) f d \mu \\
& \leq \int_{\Omega}\left(|v|^{n}+|v|^{n\left(1+\frac{1}{\delta}\right)}\right) f d \mu \\
& \leq\left[\left(\int_{\Omega}|v|^{\frac{n(1+\epsilon)}{\epsilon}} d \mu\right)^{\frac{\epsilon}{1+\epsilon}}+\left(\int_{\Omega}|v|^{\frac{n(1+\delta)(1+\epsilon)}{\delta \epsilon}} d \mu\right)^{\frac{\epsilon}{1+\epsilon}}\right]\|f\|_{L^{1+\epsilon}(\Omega)} .
\end{aligned}
$$

Combining (2.7) and (2.8), we get

$$
\int_{\Omega} f d \mu \leq A \operatorname{cap}(K, \Omega) \frac{\operatorname{cap}(K, \Omega)^{\frac{1}{\delta}}}{1+\operatorname{cap}(K, \Omega)^{\frac{1}{\delta}}},
$$

where

$$
\begin{aligned}
A & =2 C_{1} 2^{\frac{n(1+\delta)}{\delta}+2}\left(\frac{n(1+\delta)(1+\epsilon)}{\delta \epsilon}+2\right)^{\frac{n(1+\delta)}{\delta}+2}\|f\|_{L^{1+\epsilon}(\Omega)} \\
& \leq C_{2}\left(\frac{c_{0}}{\delta \epsilon}\right)^{\frac{n(1+\delta)}{\delta}+2}\|f\|_{L^{1+\epsilon}(\Omega)} .
\end{aligned}
$$

Therefore, it follows from Lemma 2.1 that

$$
-\inf _{\Omega^{\prime \prime}} u \leq C\left(\frac{c_{0}}{\delta \epsilon}\right)^{n+3 n \delta}\|f\|_{L^{1+\epsilon}(\Omega)}^{\delta}+D .
$$

Now the lemma follows from replacing $3 n \delta$ by $\delta$.

Proposition 2.1. Let $(M, g)$ be a compact Kähler manifold and let $\varphi$ be a smooth solution of the complex Monge-Ampère equation on $M$,

$$
\left\{\begin{array}{l}
\operatorname{det}\left(g_{i \bar{j}}+\varphi_{i \bar{j}}\right)=\operatorname{det}\left(g_{i \bar{j}}\right) f \\
\sup _{M} \varphi=0 .
\end{array}\right.
$$

Then, for any positive $\delta \leq \delta_{0}$ and $\epsilon \leq \epsilon_{0}$, there are two uniform constants $C, C^{\prime}$ which depend only on $g, \delta_{0}, \epsilon_{0}$ such that

$$
-\inf _{M} \varphi \leq C\left(\frac{1}{\delta \epsilon}\right)^{n+\delta}\|f\|_{L^{1+\epsilon}(M)}^{\delta}+C^{\prime} .
$$

Proof. This is a direct corollary of Lemma 2.2 (cf. the proof of Proposition 4.1 in [TZ1]). We omit its proof.

\section{A HARNACK-TYPE INEQUALity}

In this section, we use Proposition 2.1 to develop a $C^{0}$-estimate for solutions of the Monge-Ampère flow (1.2).

Proposition 3.1. Let $\varphi=\varphi_{t}$ be any solution of (1.2). Then, for any positive $\delta \leq 1$, there are two uniform constants $C=C(g, n, \delta)$ and $C^{\prime}=C^{\prime}(g, n, \delta)$ such that

$$
o s c_{M} \varphi=\sup _{M} \varphi-\inf _{M} \varphi \leq C\left(\int_{M} \varphi\left(\omega_{g}^{n}-\omega_{\varphi}^{n}\right)\right)^{n+\delta}+C^{\prime} .
$$


Proposition 3.1 is analogous to Proposition 4.2 in TZ1 for the complex MongeAmpère equation which arises from the equation for Kähler-Ricci solitons.

As before we assume that $(M, g)$ is an $n$-dimensional compact Kähler manifold such that $\omega_{g}$ represents the first Chern class $c_{1}(M)>0$. We need two lemmas in order to prove Proposition 3.1.

Lemma 3.1 (Poincaré-type inequality). Let $h$ be the smooth function determined by the relation $(1.1)$. Let $C^{\infty}(M, \mathbb{C})$ be the space of complex-valued smooth functions. Then for any $\psi \in C^{\infty}(M, \mathbb{C})$, we have

$$
\int_{M}|\bar{\partial} \tilde{\psi}|^{2} e^{h} \omega_{g}^{n} \geq \int_{M}|\tilde{\psi}|^{2} e^{h} \omega_{g}^{n}
$$

where

$$
\tilde{\psi}=\psi-\frac{1}{V} \int_{M} \psi e^{h} \omega_{g}^{n} .
$$

In particular, for any $\varphi \in C^{\infty}(M)$, we have

$$
\int_{M}|\bar{\partial} \varphi|^{2} e^{h} \omega_{g}^{n} \geq \int_{M} \varphi^{2} e^{h} \omega_{g}^{n}-\frac{1}{V}\left(\int_{M} \varphi e^{h} \omega_{g}^{n}\right)^{2} .
$$

Proof. Let $L$ be the linear differential operator on $C^{\infty}(M, \mathbb{C})$ defined by

$$
L \psi=\triangle \psi+\langle\bar{\partial} h, \overline{\partial \psi}\rangle, \quad \text { for } \quad \psi \in C^{\infty}(M, \mathbb{C}),
$$

where $\triangle$ denotes the Laplacian operator of $g$. Then $L$ is elliptic and self-adjoint with respect to the following Hermitian inner product:

$$
\left(\psi, \psi^{\prime}\right)_{h}=\int_{M} \psi \bar{\psi}^{\prime} e^{h} \omega_{g}^{n}, \quad \text { for } \quad \psi, \psi^{\prime} \in C^{\infty}(M, \mathbb{C})
$$

namely,

$$
\left(L \psi, \psi^{\prime}\right)_{h}=\left(\psi, L \psi^{\prime}\right)_{h}
$$

It follows that all eigenvalues of $L$ are real. Denote by $0=\lambda_{0}<\lambda_{1} \leq \ldots \leq \lambda_{i} \leq \ldots$ the sequence of eigenvalues of $L$ and by $\psi_{i}(i=0,1,2, \cdots)$ the corresponding sequence of eigenfunctions with the property $\left(\psi_{i}, \psi_{j}\right)_{h}=\delta_{i j}$, for any $i, j$. Note that $\psi_{0}$ is constant. Then $\left\{\psi_{i}\right\}$ is a complete orthonormal basis of the space $W^{1,2}(M, \mathbb{C})$ with respect to the weighted $L^{2}$-norm $(\cdot, \cdot)_{h}$. On the other hand, using the Bochner's technique, one can prove that $\lambda_{1} \geq 1$ ([F2]). So (3.1) holds, so does (3.2).

Lemma 3.2. Let $\varphi=\varphi_{t}$ be any solution of (1.2). Then there is a uniform constant $c_{0}>0$ independent of $t$ such that

$$
\int_{M} \exp \left\{-\frac{c_{0}}{I(\varphi)}\left(\varphi-\sup _{M} \varphi\right)\right\} \omega_{\varphi}^{n} \leq C,
$$

where $I(\varphi)=\frac{1}{V} \int_{M} \varphi\left(\omega_{g}^{n}-\omega_{\varphi}^{n}\right)>0$.

Proof. As in [TZ1], we will use an iteration argument to prove this lemma. Without loss of generality, we may assume $I(\varphi)>1$. 
Let $\bar{\varphi}=\varphi-\sup _{M} \varphi$. Then for any $p>0$, we have

$$
\begin{aligned}
\int_{M}(-\bar{\varphi})^{p}\left(\omega_{\varphi}^{n}-\omega_{\varphi}^{n-1} \wedge \omega_{g}\right) & =\frac{\sqrt{-1}}{2 \pi} \int_{M}(-\bar{\varphi})^{p} \partial \bar{\partial}(\bar{\varphi}) \wedge \omega_{\varphi}^{n-1} \\
& =p \frac{\sqrt{-1}}{2 \pi} \int_{M}(-\bar{\varphi})^{p-1}(-\partial \bar{\varphi}) \wedge(-\bar{\partial} \bar{\varphi}) \wedge \omega_{\varphi}^{n-1} \\
& =\frac{4 p}{n(p+1)^{2}} \int_{M}\left|\bar{\partial}(-\bar{\varphi})^{\frac{p+1}{2}}\right|^{2} \omega_{\varphi}^{n} .
\end{aligned}
$$

It follows that

$$
\int_{M}\left|\bar{\partial}(-\bar{\varphi})^{\frac{p+1}{2}}\right|^{2} \omega_{\varphi}^{n} \leq \frac{n(p+1)^{2}}{4 p} \int_{M}(-\bar{\varphi})^{p} \omega_{\varphi}^{n}
$$

Applying Lemma 3.1 to function $(-\bar{\varphi})^{\frac{p+1}{2}}$ in the case of the metric $\omega_{\varphi}$, we have

$$
\int_{M}\left|\bar{\partial}(-\bar{\varphi})^{\frac{p+1}{2}}\right|^{2} e^{h_{t}} \omega_{\varphi_{t}}^{n} \geq \int_{M}(-\bar{\varphi})^{p+1} e^{h_{t}} \omega_{\varphi_{t}}^{n}-\frac{1}{V}\left(\int_{M}(-\bar{\varphi})^{(p+1) / 2} e^{h_{t}} \omega_{\varphi_{t}}^{n}\right)^{2},
$$

where $h_{t}=-\frac{\partial \varphi}{\partial t}+c_{t}$ which are uniformly bounded for $t$ (cf. Lemma 1.1). Thus by using the Hölder inequality, we get

$$
\int_{M}(-\bar{\varphi})^{p+1} e^{h_{t}} \omega_{\varphi_{t}}^{n} \leq c p \int_{M}(-\bar{\varphi})^{p} e^{h_{t}} \omega_{\varphi_{t}}^{n}+\frac{1}{V} \int_{M}(-\bar{\varphi})^{p} e^{h_{t}} \omega_{\varphi_{t}}^{n} \cdot \int_{M}(-\bar{\varphi}) e^{h_{t}} \omega_{\varphi_{t}}^{n},
$$

and consequently

$$
\int_{M}(-\bar{\varphi})^{p+1} \omega_{\varphi}^{n} \leq c^{\prime}\left[p \int_{M}(-\bar{\varphi})^{p} \omega_{\varphi}^{n}+\frac{1}{V} \int_{M}(-\bar{\varphi})^{p} \omega_{\varphi}^{n} \cdot \int_{M}(-\bar{\varphi}) \omega_{\varphi}^{n}\right],
$$

where $c, c^{\prime}$ are uniform constants.

By the mean-value inequality, we have

$$
\sup _{M} \varphi \leq V^{-1} \int_{M} \varphi \omega_{g}^{n}+C .
$$

It follows that

$$
\begin{aligned}
\int_{M}(-\bar{\varphi}) \omega_{\varphi}^{n} & =V \sup _{M} \varphi+\int_{M}(-\varphi) \omega_{\varphi}^{n} \\
& \leq \int_{M} \varphi\left(\omega_{g}^{n}-\omega_{\varphi}^{n}\right)+C V \\
& \leq a I(\varphi),
\end{aligned}
$$

where $a$ is a uniform constant. Thus inserting this inequality into (3.6), we get

$$
\int_{M}(-\bar{\varphi})^{p+1} \omega_{\varphi}^{n} \leq a c^{\prime}(p+I(\varphi)) \int_{M}(-\bar{\varphi})^{p} \omega_{\varphi}^{n} .
$$

Iterating (3.7), we have

$$
\int_{M}(-\bar{\varphi})^{p+1} \omega_{\varphi}^{n} \leq 2\left(a c^{\prime} I(\varphi)\right)^{p}(p+1) ! \int_{M}(-\bar{\varphi}) \omega_{\varphi}^{n} \leq\left(c^{\prime} I(\varphi)\right)^{p+1}(p+1) !
$$


Now choosing $\varepsilon<\frac{1}{a c^{\prime} I(\varphi)}$, we obtain

$$
\begin{aligned}
\int_{M} \exp \{-\varepsilon \bar{\varphi}\} \omega_{\varphi}^{n} & =\sum_{p=0}^{+\infty} \frac{\varepsilon^{p}}{p !} \int_{M}(-\bar{\varphi})^{p} \omega_{\varphi}^{n} \\
& \leq \sum_{p=0}^{+\infty}\left(\varepsilon a c^{\prime} I(\varphi)\right)^{p} \\
& \leq \frac{1}{1-a c^{\prime} I(\varphi) \varepsilon}
\end{aligned}
$$

Put $c_{0}=\frac{1}{a c^{\prime}}$. Then (3.3) is proved.

Proof of Proposition 3.1. Let $c_{t}$ and $h_{t}$ be given as in Lemma 1.1. Let $\bar{\varphi}=\varphi-c_{t}$ and $\tilde{\varphi}=\varphi-\sup _{M} \varphi$. Then (1.2) becomes

$$
\left\{\begin{array}{l}
\operatorname{det}\left(g_{i \bar{j}}+\tilde{\varphi}_{i \bar{j}}\right)=\operatorname{det}\left(g_{i \bar{j}}\right) f, \\
\sup _{M} \tilde{\varphi}=0,
\end{array}\right.
$$

where $f=h+\frac{\partial \varphi}{\partial t}-c_{t}-\bar{\varphi}=h-h_{t}-\bar{\varphi}$. Since

$$
\int_{M} e^{h_{t}} \omega_{\varphi}^{n}=V
$$

we have

$$
0<c_{1} \leq \int_{M} e^{-\bar{\varphi}} \omega_{g}^{n} \leq c_{2}
$$

for some uniform constants $c_{1}$ and $c_{2}$. This implies

$$
\sup _{M} \bar{\varphi} \geq-C \text { and } \inf _{M} \bar{\varphi} \leq C .
$$

By (3.10) and Lemma 3.2, we have

$$
\begin{aligned}
\int_{M} \exp \left\{-\left(1+\frac{c_{0}}{I(\varphi)}\right) \bar{\varphi}\right\} \omega_{g}^{n} & \leq e^{c_{0} C} \int_{M} \exp \left\{-\frac{c_{0}}{I(\varphi)}\left(\bar{\varphi}-\sup _{M} \bar{\varphi}\right)-\bar{\varphi}\right\} \omega_{g}^{n} \\
& =e^{c_{0} C} \int_{M} \exp \left\{-\frac{c_{0}}{I(\varphi)}\left(\varphi-\sup _{M} \varphi\right)-\bar{\varphi}\right\} \omega_{g}^{n} \\
& \leq C_{1} \int_{M} \exp \left\{-\frac{c_{0}}{I(\varphi)}\left(\varphi-\sup _{M} \varphi\right)\right\} \omega_{\varphi}^{n} \leq C_{2} .
\end{aligned}
$$

It follows that

$$
\|f\|_{L^{1+\frac{c_{0}}{T(\varphi)}(M)}} \leq C_{3} .
$$

Thus, applying Proposition 2.1 to (3.8), we see that for any $\delta>0$ there are uniform constants $C_{4}$ and $C_{5}$ only depending on $\delta$ such that

$$
\sup _{M} \varphi-\inf _{M} \varphi=-\inf _{M} \tilde{\varphi} \leq C_{4} I(\varphi)^{n+\delta}+C_{5} .
$$




\section{Monotonicity of Generalized K-EnERGy}

In this section, we show the monotonicity of the generalized K-energy introduced in TZ2 along the Kähler-Ricci flow. Since $M$ has positive first Chern class, associated to any holomorphic vector field $X$ on $M$, there is a unique smooth, complex-valued function $\theta_{X}=\theta_{X}(g)$ on $M$ such that

$$
\left\{\begin{array}{l}
i_{X} \omega_{g}=\frac{\sqrt{-1}}{2 \pi} \bar{\partial} \theta_{X} \\
\int_{M} e^{\theta_{X}} \omega_{g}^{n}=\int_{M} \omega_{g}^{n}
\end{array}\right.
$$

where $i_{X} \omega_{g}$ is the interior product of $X$ with $\omega_{g}$, that is, $i_{X} \omega_{g}(Y)=\omega_{g}(X, Y)$ for any vector $Y$. The first relation above implies

$$
L_{X} \omega_{g}=d\left[i_{X}\left(\omega_{g}\right)\right]=\frac{\sqrt{-1}}{2 \pi} \partial \bar{\partial} \theta_{X} .
$$

Thus we see that $\left(g_{K S}, X\right)$ is a Kähler-Ricci soliton if and only if $h=\theta_{X}$ modulo a constant, where $h$ is a smooth function determined by the relation (1.1) associated to the metric $g_{K S}$. As an obstruction to Kähler-Ricci solitons, the following holomorphic invariant was introduced in [TZ2]:

$$
F_{X}(v)=\int_{M} v\left(h-\theta_{X}\right) e^{\theta_{X}} \omega_{g}^{n}, v \in \eta(M),
$$

where $\eta(M)$ denotes the Lie algebra consisting of all holomorphic vector fields on $M$. When $X \equiv 0$, the functional $F_{X}(\cdot)$ is just the Futaki invariant [F1]. It was shown in [TZ2] that $F_{X}(\cdot)$ is independent of the choice of Kähler metric $g$ and $F_{X}(\cdot) \equiv 0$ if there exists a Kähler-Ricci soliton $\left(g_{K S}, X\right)$ on $M$.

Let $\eta_{r}(M)$ be a reductive Lie subalgebra of $\eta(M)$ and let $X \in \eta_{r}(M)$. Let $K_{X}$ be the one-parameter group generated by $\operatorname{Im}(X)$ and let $g$ be a $K_{X}$-invariant Kähler metric. Set

$$
\mathcal{M}_{X}\left(\omega_{g}\right)=\left\{\varphi \in C^{\infty}(M) \mid \omega_{\varphi}=\omega_{g}+\frac{\sqrt{-1}}{2 \pi} \partial \bar{\partial} \varphi>0, \operatorname{Im}(X)(\varphi)=0\right\} .
$$

Associated to the invariant $F_{X}(\cdot)$, the following functional on $\mathcal{M}_{X}\left(\omega_{g}\right)$ was introduced in [TZ2]:

$$
\begin{aligned}
\tilde{\mu}_{\omega_{g}}(\varphi)= & -\frac{n}{V} \int_{0}^{1} \int_{M} \dot{\psi}\left[\operatorname{Ric}\left(\omega_{\psi}\right)-\omega_{\psi}\right. \\
& \left.-\frac{\sqrt{-1}}{2 \pi}\left(\partial \bar{\partial} \theta_{X}\left(\omega_{\psi}\right)-\partial\left(h_{\omega_{\psi}}-\theta_{X}\left(\omega_{\psi}\right)\right) \wedge \bar{\partial} \theta_{X}\left(\omega_{\psi}\right)\right)\right] \wedge e^{\theta_{X}\left(\omega_{\psi}\right)} \omega_{\psi}^{n-1} \wedge d t,
\end{aligned}
$$

where $\psi=\psi_{t}(0 \leq t \leq 1)$ is a path connecting 0 to $\varphi$ in $\mathcal{M}_{X}\left(\omega_{g}\right), \theta_{X}\left(\omega_{\psi}\right)=$ $\theta_{X}+X(\psi)$ and $\operatorname{Ric}\left(\omega_{\psi}\right)$ denotes the Ricci form of $\omega_{\psi}$. If $X=0$, then $\tilde{\mu}_{\omega_{g}}(\varphi)$ is just Mabuchi's $K$-energy $\mathrm{Ma}$.

Let $X$ be in the center of $\eta_{r}(M)$ and let $\operatorname{Aut}_{r}(M)$ be the connected subgroup associated to $\eta_{r}(M)$. Then

$$
\rho^{*} L_{X} \omega_{\varphi}=L_{X}\left(\rho^{*} \omega_{\varphi}\right), \forall \rho \in \operatorname{Aut}_{r}(M) .
$$

Thus if $\varphi \in \mathcal{M}_{X}\left(\omega_{g}\right)$, then

$$
\varphi_{\rho} \in \mathcal{M}_{X}\left(\omega_{g}\right), \forall \rho \in \operatorname{Aut}_{r}(M),
$$

where $\varphi_{\rho}$ is the Kähler potential function determined by

$$
\rho^{*} \omega_{\varphi}=\omega_{g}+\frac{\sqrt{-1}}{2 \pi} \partial \bar{\partial} \varphi_{\rho}
$$


Moreover, analogous to the case of the $K$-energy and the Futaki invariant, we have the following for $\tilde{\mu}_{\omega_{g}}$ :

$$
\frac{d \tilde{\mu}_{\omega_{g}}\left(\varphi_{\rho_{t}}\right)}{d t}=\operatorname{Re}\left(F_{X}(v)\right)
$$

where $\rho_{t}=\exp \{t v\}$ is a one-parameter subgroup generated by a holomorphic vector field $v$ on $M$. This implies that if $F_{X}(\cdot) \equiv 0$, then

$$
\tilde{\mu}_{\omega_{g}}\left(\varphi_{\rho}\right)=\tilde{\mu}_{\omega_{g}}(\varphi), \forall \rho \in \operatorname{Aut}_{r}(M) .
$$

Note that $X$ must lie in the center of $\eta_{r}(M)$ if $F_{X}(\cdot) \equiv 0$ [TZ1].

Lemma 4.1. Let $\varphi$ be a solution of the flow (1.2). Suppose that the initial metric $g$ is $K_{X}$-invariant and that the holomorphic invariant $F_{X}($.$) vanishes. Then$

$$
\tilde{\mu}_{\omega_{g}}(\varphi) \leq 0 .
$$

Proof. Let $\sigma_{t}=\exp \{t X\}$ be a one-parameter subgroup in $\operatorname{Aut}_{r}(M)$ generated by $X$ and let $\varphi^{\prime}=\varphi_{\sigma_{t}}$ be defined as above for the subgroup $\left\{\sigma_{t}\right\}$. Then $\omega_{\varphi^{\prime}}$ satisfies the modified Kähler-Ricci flow,

$$
\frac{\partial}{\partial t} \omega_{\varphi^{\prime}}=-\operatorname{Ric}\left(\omega_{\varphi^{\prime}}\right)+\omega_{\varphi^{\prime}}+L_{X} \omega_{\varphi^{\prime}}
$$

Then, using integration by parts, we have

$$
\frac{d \tilde{\mu}_{\omega_{g}}\left(\varphi^{\prime}\right)}{d t}=-\frac{1}{V} \int_{M}\left\|\bar{\partial} \frac{\partial \varphi^{\prime}}{\partial t}\right\|^{2} e^{\theta_{X}+X\left(\varphi^{\prime}\right)}\left(\omega_{\varphi^{\prime}}\right)^{n} \leq 0 .
$$

It follows that

$$
\tilde{\mu}_{\omega_{g}}\left(\varphi^{\prime}\right) \leq 0,
$$

and consequently, by (4.2), we get (4.3).

It was shown in TZ2 that if $M$ admits a Kähler-Ricci soliton $\left(g_{K S}, X\right)$, then the functional $\tilde{\mu}_{\omega_{g}}(\cdot)$ is bounded from below on $\mathcal{M}_{X}\left(\omega_{g}\right)$. So by $(4.5)$, we have

$$
\frac{1}{V} \int_{0}^{\infty} \int_{M}\left\|\bar{\partial} \frac{\partial \varphi^{\prime}}{\partial t}\right\|^{2} e^{\theta_{X}+X\left(\varphi^{\prime}\right)}\left(\omega_{\varphi^{\prime}}\right)^{n} \wedge d t \leq C .
$$

It follows that

$$
\frac{1}{V} \int_{0}^{\infty} \int_{M}\left\|\bar{\partial} \frac{\partial \varphi^{\prime}}{\partial t}\right\|^{2} e^{\theta_{X}+X\left(\varphi^{\prime}\right)-t}\left(\omega_{\varphi^{\prime}}\right)^{n} \wedge d t \leq C .
$$

By using the Maximal Principle, we get from (4.4) that

$$
\frac{\partial \varphi^{\prime}}{\partial t}=\log \frac{\operatorname{det}\left(g_{i \bar{j}}+\varphi_{i \bar{j}}^{\prime}\right)}{\operatorname{det}\left(g_{i \bar{j}}\right)}+X\left(\varphi^{\prime}\right)+\varphi^{\prime}-h+\theta_{X} .
$$

Differentiating the above equation on $t$, we obtain

$$
\frac{\partial}{\partial t} \frac{\partial \varphi^{\prime}}{\partial t}=\triangle^{\prime} \frac{\partial \varphi^{\prime}}{\partial t}+X\left(\frac{\partial \varphi^{\prime}}{\partial t}\right)+\frac{\partial \varphi^{\prime}}{\partial t} .
$$

Let

$$
a_{t}=\int_{M} \frac{\partial \varphi^{\prime}}{\partial t} e^{\theta X+X\left(\varphi^{\prime}\right)} \omega_{\varphi^{\prime}}^{n} .
$$

Then it is easy to see that

$$
\frac{d a_{t}}{d t}=a_{t}-\int_{M}\left\|\bar{\partial} \frac{\partial \varphi^{\prime}}{\partial t}\right\|^{2} e^{\theta X+X\left(\varphi^{\prime}\right)}\left(\omega_{\varphi^{\prime}}\right)^{n} .
$$


Lemma 4.2. Suppose that $M$ admits a Kähler-Ricci soliton $\left(g_{K S}, X\right)$. Let $g$ be a $K_{X}$-invariant Kähler metric on $M$. We choose $h$ in equation (1.2) by adding a suitable constant so that

$$
\frac{1}{V} \int_{M}\left(h-\theta_{X}\right) \omega_{g}^{n}=-\frac{1}{V} \int_{0}^{\infty} \int_{M}\left\|\bar{\partial} \frac{\partial \varphi^{\prime}}{\partial t}\right\|^{2} e^{\theta_{X}+X\left(\varphi^{\prime}\right)-t}\left(\omega_{\varphi^{\prime}}\right)^{n} \wedge d t .
$$

Then the solution $\varphi^{\prime}(t, \cdot)$ of $(4.7)$ with the initial condition $\varphi^{\prime}(0, \cdot)=0$ satisfies

$$
\lim _{t \rightarrow \infty} \int_{M} \frac{\partial \varphi^{\prime}}{\partial t} e^{\theta X+X\left(\varphi^{\prime}\right)} \omega_{\varphi^{\prime}}^{n}=0 .
$$

Proof. By (4.9), we have

$$
\frac{\left(e^{-t} a_{t}\right)}{d t}=-\int_{M} e^{-t}\left\|\bar{\partial} \frac{\partial \varphi^{\prime}}{\partial t}\right\|^{2} e^{\theta_{X}+X\left(\varphi^{\prime}\right)}\left(\omega_{\varphi^{\prime}}\right)^{n} .
$$

Note that

and

$$
h-\theta_{X}=-\left.\frac{\partial \varphi^{\prime}}{\partial t}\right|_{t=0}
$$

Then we have

$$
a_{0}=\int_{0}^{\infty} \int_{M}\left\|\bar{\partial} \frac{\partial \varphi^{\prime}}{\partial t}\right\|^{2} e^{\theta_{X}+X\left(\varphi^{\prime}\right)-t}\left(\omega_{\varphi^{\prime}}\right)^{n} \wedge d t .
$$

$$
\begin{aligned}
a_{t} & =\int_{t}^{\infty} e^{t-s}\left\|\bar{\partial} \frac{\partial \varphi^{\prime}}{\partial t}\right\|^{2} e^{\theta_{X}+X\left(\varphi^{\prime}\right)}\left(\omega_{\varphi^{\prime}}\right)^{n} \wedge d s \\
& \leq \int_{t}^{\infty}\left\|\bar{\partial} \frac{\partial \varphi^{\prime}}{\partial t}\right\|^{2} e^{\theta_{X}+X\left(\varphi^{\prime}\right)}\left(\omega_{\varphi^{\prime}}\right)^{n} \wedge d s \\
& \rightarrow 0, \text { as } t \rightarrow \infty .
\end{aligned}
$$

Thus the lemma is true.

Proposition 4.1. Suppose that $M$ admits a Kähler-Ricci soliton $\left(g_{K S}, X\right)$. Let $\varphi$ be a solution of (1.2). Suppose that the initial metric $g$ is $K_{X}$-invariant and that $h$ is normalized as in Lemma 4.2. Then the constants $c_{t}$ in Lemma 1.1 are uniformly bounded. Consequently, the $\frac{\partial \varphi}{\partial t}$ are uniformly bounded.

Proof. Let $\varphi^{\prime}(t, \cdot)$ be the solution of equation (4.7). Then

$$
\frac{\partial \varphi^{\prime}}{\partial t}=\frac{\partial \varphi}{\partial t}+\theta_{X}+X\left(\varphi^{\prime}\right)
$$

Thus by Lemma 4.2, we get

$$
\left|\int_{M}-\frac{\partial \varphi}{\partial t} e^{\theta_{X}+X\left(\varphi^{\prime}\right)} \omega_{\varphi^{\prime}}^{n}\right| \leq C
$$

for some uniform constant $C$, and so

$$
V\left|c_{t}\right| \leq C+\left|\int_{M} h_{t} e^{\theta_{X}+X\left(\varphi^{\prime}\right)} \omega_{\varphi^{\prime}}^{n}\right| .
$$

Here we use the fact that the $|X(\psi)|$ are uniformly bounded for any $\psi \in \mathcal{M}_{X}\left(\omega_{g}\right)$ [Z]. Therefore by Lemma 1.1, we obtain

and

$$
\left|c_{t}\right| \leq \frac{C}{V}+A
$$

$$
\left|\frac{\partial \varphi}{\partial t}\right| \leq \frac{C}{V}+2 A .
$$




\section{A $C^{0}$-Estimate}

In this section, we use the properness and monotonicity of $\tilde{\mu}_{\omega_{g}}(\cdot)$ to derive the $C^{0}$-estimate for solutions of (1.2). We will assume that $M$ admits a Kähler-Ricci soliton $\left(g_{K S}, X\right)$.

Let us recall two other functionals on $\mathcal{M}_{X}\left(\omega_{g}\right)$ introduced in [TZ2]:

$$
\begin{aligned}
\tilde{F}_{\omega_{g}}(\varphi) & =\tilde{J}(\varphi)-\frac{1}{V} \int_{M} \varphi e^{\theta_{X}} \omega_{g}^{n}-\log \left(\frac{1}{V} \int_{M} e^{h-\varphi} \omega_{g}^{n}\right) \\
& =-\frac{1}{V} \int_{0}^{1} \int_{M} \frac{\partial \psi}{\partial t} e^{\theta_{X}+X(\psi)} \omega_{\psi}^{n} \wedge d t-\log \left(\frac{1}{V} \int_{M} e^{h-\varphi} \omega_{g}^{n}\right)
\end{aligned}
$$

and

$$
\tilde{J}(\varphi)=\frac{1}{V} \int_{0}^{1} \int_{M} \frac{\partial \psi}{\partial t}\left(e^{\theta_{X}} \omega_{g}^{n}-e^{\theta_{X}+X(\psi)} \omega_{\psi}^{n}\right) \wedge d t
$$

where $\psi=\psi_{t}(0 \leq t \leq 1)$ is any path from 0 to $\varphi$ and both functionals are independent of the choice of path [Zh]. The following result was proved in [TZ2].

Lemma 5.1. We have

$\tilde{\mu}_{\omega_{g}}(\varphi)=\tilde{F}_{\omega_{g}}(\varphi)-\frac{1}{V} \int_{M}\left(h_{\omega_{\varphi}}-\theta_{X}-X(\varphi)\right) e^{\theta_{X}+X(\varphi)} \omega_{\varphi}^{n}+\frac{1}{V} \int_{M}\left(h-\theta_{X}\right) e^{\theta_{X}} \omega_{g}^{n}$,

where $h$ and $h_{\omega_{\varphi}}$ are smooth functions determined by the relation (1.1) associated to $\omega_{g}$ and $\omega_{\varphi}$, respectively, and normalized by

$$
\int_{M} e^{h} \omega_{g}^{n}=\int_{M} e^{h_{\varphi}} \omega_{\varphi}^{n}=V
$$

In particular, we have

$$
\tilde{\mu}_{\omega_{g}}(\varphi) \geq \tilde{F}_{\omega_{g}}(\varphi)-C
$$

The following lemma was proved in [CTZ] by using the same arguments in [TZ1].

Lemma 5.2. Assume that $M$ admits a Kähler-Ricci soliton $\left(g_{K S}, X\right)$, and let $\Lambda_{1}\left(\omega_{K S}, X\right)$ be the space of eigenfunctions of the first nonzero eigenvalue of the second order elliptic operator $L$ defined on $\mathcal{M}_{X}\left(\omega_{K S}\right)$, where

$$
L \varphi=\triangle_{g_{K S}} \varphi+X(\varphi) .
$$

Then for any $\varphi \in \Lambda_{1}\left(X, g_{K S}\right)^{\perp}$, we have

$$
\tilde{F}_{\omega_{K S}}(\varphi) \geq C I_{\omega_{K S}}(\varphi)^{\frac{1}{4 n+5}}-C^{\prime}
$$

for some uniform constants $C$ and $C^{\prime}$, where

$$
I_{\omega_{K S}}(\varphi)=\frac{1}{V} \int_{M} \varphi\left(\omega_{K S}^{n}-\left(\omega_{K S}+\frac{\sqrt{-1}}{2 \pi} \partial \bar{\partial} \varphi\right)^{n}\right) .
$$

In order to apply the above lemma to obtain a $C^{0}$-estimate for solutions of (1.2), we shall modify the flow. Let $\rho_{t} \in \operatorname{Aut}_{r}(M)$ be a family of holomorphic transformations on $M$. Let

$$
\omega_{\tilde{\varphi}}=\rho_{t}^{*} \omega_{\varphi}=\omega_{g}+\frac{\sqrt{-1}}{2 \pi} \partial \bar{\partial} \tilde{\varphi},
$$

where $\omega_{\varphi}$ is the evolved metrics in the flow (1.2). Then $\omega_{\tilde{\varphi}}$ satisfies

$$
\frac{\partial}{\partial t} \omega_{\tilde{\varphi}}=-\operatorname{Ric}\left(\omega_{\tilde{\varphi}}\right)+\omega_{\tilde{\varphi}}+L_{\operatorname{Re}(\tilde{X})} \omega_{\tilde{\varphi}},
$$


where $\tilde{X}=\tilde{X}_{t}=\rho_{t}^{-1} \cdot \frac{d \rho_{t}}{d t} \in \eta(M)$ is a family of holomorphic vector fields on $M$. Consequently we get a Monge-Ampère flow for $\tilde{\varphi}$ (modulo a constant),

$$
\frac{\partial \tilde{\varphi}}{\partial t}=\log \frac{\operatorname{det}\left(g_{i \bar{j}}+\tilde{\varphi}_{i \bar{j}}\right)}{\operatorname{det}\left(g_{i \bar{j}}\right)}+\operatorname{Re}(\tilde{X}(\tilde{\varphi}))+\tilde{\varphi}-h+\operatorname{Re}\left(\theta_{\tilde{X}}\right),
$$

where $\theta_{\tilde{X}}$ is the smooth function defined by (4.1) for the holomorphic vector field $\tilde{X}$. From the proof of the uniqueness of Kähler-Ricci solitons in [TZ1, one can choose a holomorphic transformation $\rho_{t}$ for each $t$ so that $\tilde{\varphi}(t, \cdot)-\Psi_{0} \in \Lambda_{1}\left(\omega_{K S}, X\right)^{\perp}$, where $\Psi_{0}$ is a smooth function satisfying

$$
\omega_{g}=\omega_{K S}-\frac{\sqrt{-1}}{2 \pi} \partial \bar{\partial} \Psi_{0}
$$

Proposition 5.1. Suppose that $M$ admits a Kähler-Ricci soliton $\left(g_{K S}, X\right)$. Let $\tilde{\varphi}$ be a solution of (5.6). Suppose that the initial metric $g$ is $K_{X}$-invariant and that $h$ is normalized as in Lemma 4.2. Then there is a uniform constant $C$ such that

$$
\|\tilde{\varphi}\|_{C^{0}(M)} \leq C .
$$

Proof. Note that the existence of Kähler-Ricci soliton $\left(g_{K S}, X\right)$ implies that $F_{X}(\cdot) \equiv$ 0 . By (4.3) in Lemma 4.1, we have

$$
\tilde{\mu}_{\omega_{g}}(\tilde{\varphi})=\tilde{\mu}_{\omega_{g}}(\varphi) \leq 0
$$

It follows that

$$
\begin{aligned}
\tilde{\mu}_{\omega_{K S}}\left(\tilde{\varphi}-\Psi_{0}\right) & =\tilde{\mu}_{\omega_{K S}}\left(-\Psi_{0}\right)+\tilde{\mu}_{\omega_{g}}(\tilde{\varphi}) \\
& \leq \tilde{\mu}_{g_{K S}}\left(-\Psi_{0}\right) .
\end{aligned}
$$

On the other hand, by Lemma 5.2, we have

$$
\tilde{F}_{\omega_{K S}}\left(\tilde{\varphi}-\Psi_{0}\right) \geq C I_{\omega_{K S}}\left(\tilde{\varphi}-\Psi_{0}\right)^{\frac{1}{4 n+5}}-C^{\prime} .
$$

Thus by Lemma 5.1, we see that there is a uniform constant $C_{1}$ such that

$$
I_{\omega_{K S}}\left(\tilde{\varphi}-\Psi_{0}\right) \leq C_{1} .
$$

This shows that

$$
I(\tilde{\varphi}) \leq C_{2}
$$

It is clear that (5.6) is equal to

$$
\operatorname{det}\left(g_{i \bar{j}}+\tilde{\varphi}_{i \bar{j}}\right)=\operatorname{det}\left(g_{i \bar{j}}\right) \exp \left\{h+\frac{\partial \varphi}{\partial t} \circ \rho_{t}-\tilde{\varphi}\right\} .
$$

Since $\frac{\partial \varphi}{\partial t} \circ \rho_{t}$ is uniformly bounded (Proposition 4.1), we can apply Proposition 3.1 to the solution $\tilde{\varphi}$ of (5.10). Thus by (5.9), we have

$$
\operatorname{osc}_{M} \tilde{\varphi} \leq C_{3}\left(I(\tilde{\varphi})^{n+1}+1\right) \leq C_{4} .
$$

The estimate (5.8) follows from this. 


\section{Higher ORDER ESTIMATES AND PROOF OF MAIN THEOREM}

In order to get higher order estimates of solutions to (5.6), we shall modify the gauge transformations again which appeared in Section 5 . The argument used here is similar to one in Sections 6 and 7 in [CT2. First we observe

Lemma 6.1. Let $\rho_{t}$ be a family of holomorphic transformations defined in Section 5. Then for each integer $i=0,1, \ldots$, we have

$$
\left\|\rho_{i+1}^{-1} \cdot \rho_{i}-I\right\| \leq C
$$

for some uniform constant $C$, where the norm $\|\cdot\|$ denotes the distance between two elements in Aut $(M)$.

Proof. Let $\tilde{\varphi}$ be a solution of (5.6). Then

$$
\left(\rho_{i}\right)^{*} \omega_{\varphi_{i}}=\omega_{\tilde{\varphi}_{i}}=\omega_{g}+\frac{\sqrt{-1}}{2 \pi} \partial \bar{\partial} \tilde{\varphi}_{i}
$$

and

$$
\left(\rho_{i+1}\right)^{*} \omega_{\varphi_{i+1}}=\omega_{\tilde{\varphi}_{i+1}}=\omega_{g}+\frac{\sqrt{-1}}{2 \pi} \partial \bar{\partial} \tilde{\varphi}_{i+1} .
$$

It follows that

$$
\begin{aligned}
\left(\rho_{i}\right)^{*} \omega_{\varphi_{i+1}}-\left(\rho_{i}\right)^{*} \omega_{\varphi_{i}} & =\left(\rho_{i+1} \cdot\left(\rho_{i+1}^{-1} \cdot \rho_{i}\right)\right)^{*} \omega_{\varphi_{i+1}}-\left(\rho_{i}\right)^{*} \omega_{\varphi_{i}} \\
& =\frac{\sqrt{-1}}{2 \pi} \partial \bar{\partial}\left[\tilde{\varphi}_{i+1} \cdot\left(\rho_{i+1}^{-1} \rho_{i}\right)-\tilde{\varphi}_{i}\right]+\left[\left(\rho_{i+1}^{-1} \rho_{i}\right)^{*} \omega_{g}-\omega_{g}\right] .
\end{aligned}
$$

Thus

$$
\left(\rho_{i+1}^{-1} \rho_{i}\right)^{*} \omega_{g}-\omega_{g}=-\frac{\sqrt{-1}}{2 \pi} \partial \bar{\partial}\left[\tilde{\varphi}_{i+1} \cdot\left(\rho_{i+1}^{-1} \rho_{i}\right)-\tilde{\varphi}_{i}+\left(\int_{i}^{i+1} \frac{\partial \varphi}{\partial t} d t\right) \cdot \rho_{i}\right] .
$$

Since the smooth function

$$
G=\tilde{\varphi}_{i+1} \cdot\left(\rho_{i+1}^{-1} \rho_{i}\right)-\tilde{\varphi}_{i}+\left(\int_{i}^{i+1} \frac{\partial \varphi}{\partial t} d t\right) \cdot \rho_{i}
$$

is uniformly bounded by Proposition 4.1 and (5.1), we have

$$
\left\|\rho_{i+1}^{-1} \rho_{i}-I\right\| \leq C
$$

for some uniform constant $C$.

Using Lemma 6.1, one can choose a modified family of holomorphic transformations $\bar{\rho}_{t} \in \operatorname{Aut}_{r}(M)(0<t<\infty)$ to replace $\rho_{t}$ such that for any $t \in(0, \infty)$ (cf. CT2]),

$$
\left\|\rho_{t}^{-1} \bar{\rho}_{t}-I d\right\| \leq C
$$

and

$$
\left\|\left(\bar{\rho}_{t}^{-1}\right)_{*} \frac{d \bar{\rho}_{t}}{d t}\right\|_{g} \leq C
$$

where the $\left(\bar{\rho}_{t}^{-1}\right)_{*} \frac{d \bar{\rho}_{t}}{d t}=\bar{X}_{t} \in \eta_{r}(M)$ induce a family of holomorphic vector fields on $M$. Furthermore, for any $k \geq 0$, we may assume that there is a constant $C_{k}$ such that

$$
\left\|\frac{\partial^{k} \bar{X}_{t}}{\partial t^{k}}\right\|_{g} \leq C_{k} .
$$


Let $\bar{\varphi}=\bar{\varphi}_{t}$ be a family of smooth functions determined by

$$
\left(\bar{\rho}_{t}\right)^{*} \omega_{\varphi}=\omega_{g}+\frac{\sqrt{-1}}{2 \pi} \partial \bar{\partial} \bar{\varphi} .
$$

Such a family is unique modulo addition by constants. Then by adding appropriate constants if needed, we may assume that $\bar{\varphi}$ satisfies a complex Monge-Ampère equation

$$
\operatorname{det}\left(g_{i \bar{j}}+\bar{\varphi}_{i \bar{j}}\right)=\operatorname{det}\left(g_{i \bar{j}}\right) \exp \left\{h+\frac{\partial \varphi}{\partial t} \cdot \bar{\rho}-\bar{\varphi}\right\}
$$

Since

$$
\frac{\sqrt{-1}}{2 \pi} \partial \bar{\partial} \bar{\varphi}=\left(\rho_{t}^{-1} \bar{\rho}_{t}-I\right)^{*} \omega_{g}+\frac{\sqrt{-1}}{2 \pi} \partial \bar{\partial}\left(\left(\rho_{t}^{-1} \bar{\rho}_{t}\right)^{*} \tilde{\varphi}\right),
$$

Proposition 5.1 implies that

$$
\|\bar{\varphi}\|_{C^{0}(M)} \leq C
$$

for some uniform $C$.

To obtain a $C^{2}$-estimate and $C^{3}$-estimate for $\bar{\varphi}$, we need to further modify the Kähler-Ricci flow equation. Let $\sigma_{t}=\exp \{t X\}$ be an one-parameter subgroup generated by $X$ associated to the Kähler-Ricci soliton $\omega_{K S}$ and let $\omega_{\rho}=$ $\left(\bar{\rho}_{t}^{-1} \cdot \sigma_{t}\right)^{*} \omega_{K S}$. Define $f_{\rho}=f_{\rho_{t}}$ by

$$
\left(\bar{\rho}_{t}^{-1} \cdot \sigma_{t}\right)^{*} \omega_{K S}=\omega_{K S}+\frac{\sqrt{-1}}{2 \pi} \partial \bar{\partial} f_{\rho} .
$$

Since $\left(\bar{\rho}_{t}^{-1} \cdot \sigma_{t}\right)^{*} \omega_{K S}$ is also a Kähler-Ricci soliton w.r.t to $X$, we may assume that $f_{\rho}$ modulo a constant satisfies the equation

$$
\operatorname{det}\left(g_{i \bar{j}}+\left(f_{\rho}+\Psi_{0}\right)_{i \bar{j}}\right)=\operatorname{det}\left(g_{i \bar{j}}\right) e^{h-\theta_{X}-X\left(f_{\rho}+\Psi_{0}\right)-f_{\rho}-\Psi_{0}},
$$

where $\Psi_{0}$ is given as in (5.7). Differentiating the equation on $t$, we have

$$
\Delta_{\rho} \frac{\partial f_{\rho}}{\partial t}+\frac{\partial f_{\rho}}{\partial t}=-X\left(\frac{\partial f_{\rho}}{\partial t}\right)
$$

where $\Delta_{\rho}=\Delta_{\rho_{t}}$ is the Laplacian operator of the metric $\omega_{\rho}$. This implies

$$
\frac{\partial f_{\rho}}{\partial t}=\left.\left(\bar{\rho}_{0} \bar{\rho}_{t}^{-1} \cdot \sigma_{t}\right)^{*} \frac{\partial f_{\rho}}{\partial t}\right|_{t=0} .
$$

On the other hand, $\frac{\partial f_{\rho}}{\partial t}$ satisfies

$$
L_{X-\bar{X}}\left(\omega_{\rho_{t}}\right)=\frac{\sqrt{-1}}{2 \pi} \partial \bar{\partial} \frac{\partial f_{\rho}}{\partial t} .
$$

It follows that modulo constants, we have

$$
\frac{\partial f_{\rho}}{\partial t}=\theta_{X-\bar{X}}\left(\omega_{\rho_{t}}\right)
$$

where $\theta_{X-\bar{X}}\left(\omega_{\rho_{t}}\right)$ is determined by

$$
\left\{\begin{array}{l}
i_{X-\bar{X}}\left(\omega_{\rho_{t}}\right)=\frac{\sqrt{-1}}{2 \pi} \bar{\partial} \theta_{X-\bar{X}}\left(\omega_{\rho_{t}}\right) \\
\int_{M} e^{\theta_{X-X}\left(\omega_{\rho_{t}}\right)} \omega_{\rho_{t}}^{n}=V .
\end{array}\right.
$$

Observing that

$$
\int_{M} \frac{\partial f_{\rho}}{\partial t} e^{\theta_{X}\left(\omega_{\rho_{t}}\right)} \omega_{\rho_{t}}^{n}=0
$$


we can deduce

$$
\left|\theta_{X-\bar{X}}\left(\omega_{\rho_{t}}\right)-\frac{\partial f_{\rho}}{\partial t}\right| \leq C_{0}
$$

for some uniform constant $C_{0}$. In particular,

$$
\left\|\left.\frac{\partial f_{\rho}}{\partial t}\right|_{t=0}\right\|_{C^{0}(M)} \leq\left\|\left.\theta_{X-\bar{X}}\left(\omega_{\rho_{t}}\right)\right|_{t=0}\right\|_{C^{0}(M)}+C_{0} .
$$

Combining this with (6.7), we get

$$
\left\|\frac{\partial f_{\rho}}{\partial t}\right\|_{C^{0}(M)} \leq C
$$

Moreover, for any integer $l \geq 0$, one can have

$$
\left\|\nabla_{\rho}^{l} \frac{\partial f_{\rho}}{\partial t}\right\|_{\omega_{\rho}} \leq C
$$

where $\nabla_{\rho}^{l}$ denotes the $l$-th covariant derivative with respect to the metric $\omega_{\rho}$.

Let $\psi=\psi_{t}=\left(\bar{\rho}_{t}^{-1} \cdot \sigma_{t}\right)^{*}\left(\bar{\varphi}-\Psi_{0}\right)$. Then $\psi$ is uniformly bounded. Note that $\psi$ satisfies

$$
\begin{aligned}
\omega_{\varphi^{\prime}} & =\left(\bar{\rho}^{-1} \sigma\right)^{*} \omega_{K S}+\frac{\sqrt{-1}}{2 \pi} \partial \bar{\partial} \psi \\
& =\omega_{g}+\frac{\sqrt{-1}}{2 \pi} \partial \bar{\partial}\left(\psi+f_{\rho}+\Psi_{0}\right),
\end{aligned}
$$

where $\omega_{\varphi^{\prime}}$ is defined as in Section 4. Thus by (4.4) and (6.6), we get a parabolic equation for $\psi$ :

$$
\frac{\partial \psi}{\partial t}=\log \frac{\omega_{\varphi^{\prime}}^{n}}{\omega_{\rho}^{n}}+X(\psi)+\psi-\frac{\partial f_{\rho}}{\partial t} .
$$

This is equivalent to

$$
\frac{\partial \psi}{\partial t}=\log \frac{\left(\omega_{\rho}+\frac{\sqrt{-1}}{2 \pi} \partial \bar{\partial} \psi\right)^{n}}{\omega_{\rho}^{n}}+X(\psi)+\psi-\frac{\partial f_{\rho}}{\partial t} .
$$

Since

and

$$
\frac{\left(\omega_{\rho}+\frac{\sqrt{-1}}{2 \pi} \partial \bar{\partial} \psi\right)^{n}}{\omega_{\rho}^{n}}=\left(\bar{\rho}_{t}^{-1} \cdot \sigma_{t}\right)^{*}\left(\frac{\omega \frac{n}{\varphi}}{\omega_{K E}^{n}}\right)
$$

we have

$$
\begin{aligned}
\left|\log \frac{\omega_{\frac{\varphi}{\varphi}}^{n}}{\omega_{K S}^{n}}\right| & =\left|\log \frac{\omega_{\bar{\varphi}}^{n}}{\omega_{g}^{n}}+\log \frac{\omega_{g}^{n}}{\omega_{K S}^{n}}\right| \\
& =\left|h+\frac{\partial \varphi}{\partial t} \cdot \bar{\rho}-\bar{\varphi}+\log \frac{\omega_{g}^{n}}{\omega_{K S}^{n}}\right| \leq C,
\end{aligned}
$$

$$
\left|\log \left(\frac{\left(\omega_{\rho}+\frac{\sqrt{-1}}{2 \pi} \partial \bar{\partial} \psi\right)^{n}}{\omega_{\rho}^{n}}\right)\right| \leq C
$$

It follows that

$$
\left|\frac{\partial \psi}{\partial t}\right| \leq\left|\log \left(\frac{\left(\omega_{\rho}+\frac{\sqrt{-1}}{2 \pi} \partial \bar{\partial} \psi\right)^{n}}{\omega_{\rho}^{n}}\right)\right|+|X(\psi)|+|\psi|+\left|\frac{\partial f_{\rho}}{\partial t}\right| \leq C^{\prime} .
$$

Here we have used the fact that the $|X(\psi)|$ are uniformly bounded for all $\psi \in$ $\mathcal{M}_{X}\left(\omega_{g}\right)$ Zh]. 


\section{Lemma 6.2.}

$$
n+\Delta_{\rho} \psi \leq C .
$$

Proof. Let $\Delta^{\prime}=\Delta_{t}^{\prime}$ be the Laplacian operator associated to $\omega_{\varphi_{t}^{\prime}}$. Set

$$
F=\frac{\partial \psi}{\partial t}-X(\psi)-\psi+\frac{\partial f_{\rho}}{\partial t}
$$

Then for sufficiently large $c$, using (6.9) and following arguments in Ya, we compute

$$
\begin{aligned}
\Delta^{\prime}\left(e^{-c \psi}\left(n+\Delta_{\rho} \psi\right)\right) \geq e^{-c \psi}[\Delta F- & C_{1}-\operatorname{cn}\left(n+\Delta_{\rho} \psi\right) \\
& \left.+\frac{c}{2}\left(n+\Delta_{\rho} \psi\right) \sum_{i=1}^{n} \frac{1}{1+\psi_{i \bar{i}}}\right],
\end{aligned}
$$

where we choose a local coordinate system at a given point $p \in M$ so that $\omega_{\rho}=$ $\frac{\sqrt{-1}}{2 \pi} \sum_{i=1}^{n} d z^{i} \wedge d \bar{z}^{i}$ at $p$ and $C_{1}$ depends only on the metric $\omega_{K S}$. Note that

$$
\begin{aligned}
\Delta_{\rho} F & =\Delta_{\rho} \frac{\partial \psi}{\partial t}-\left(n+\Delta_{\rho} \psi\right)+n-\Delta_{\rho}(X(\psi))+\Delta_{\rho} \frac{\partial f_{\rho}}{\partial t} \\
& \geq \frac{\partial}{\partial t}\left(n+\Delta_{\rho} \psi\right)-\left(\left\|\nabla_{\rho} X\right\|_{\omega_{\rho}}+1\right)\left(n+\Delta_{\rho} \psi\right)-c\left(n+\Delta_{\rho} \psi\right) \sup _{M} X(\psi) \\
& -e^{c \psi}\left(X\left(e^{-c \psi}\left(n+\Delta_{\rho} \psi\right)\right)\right)-\sum_{i=1}^{n}\left(\frac{\partial f_{\rho}}{\partial t}\right)_{i \bar{i}} \psi_{i \bar{i}}+\Delta_{\rho} \frac{\partial f_{\rho}}{\partial t}
\end{aligned}
$$

and

$$
\left\|\nabla_{\rho} X\right\|_{\omega_{\rho}}=\|\nabla X\|_{\omega_{K S}} .
$$

Thus by (6.8), we get

$$
\begin{aligned}
\Delta_{\rho} F & \geq \frac{\partial}{\partial t}\left(n+\Delta_{\rho} \psi\right)-C_{2}\left(n+\Delta_{\rho} \psi\right)-C_{3} \\
& -e^{c \psi}\left(X\left(e^{-c \psi}\left(n+\Delta_{\rho} \psi\right)\right)\right) .
\end{aligned}
$$

Since

$$
\begin{aligned}
\sum_{i=1}^{n} \frac{1}{1+\psi_{i \bar{i}}} & \geq \prod_{i=1}^{n}\left(1+\psi_{i \bar{i}}\right)^{-\frac{1}{n-1}}\left(n+\Delta_{\rho} \psi\right)^{\frac{1}{n-1}} \\
& =e^{-\frac{F}{n-1}}\left(n+\Delta_{\rho} \psi\right)^{\frac{1}{n-1}} \\
& \geq C_{4} e^{\frac{\psi}{n-1}}\left(n+\Delta_{\rho} \psi\right)^{\frac{1}{n-1}}
\end{aligned}
$$

and

$$
\begin{aligned}
\frac{\partial}{\partial t}\left(e^{-c \psi}\left(n+\Delta_{\rho} \psi\right)\right) & =e^{-c \psi} \frac{\partial}{\partial t}\left(n+\Delta_{\rho} \psi\right)-c \frac{\partial \psi}{\partial t}\left(n+\Delta_{\rho} \psi\right) \\
& \leq e^{-c \psi} \frac{\partial}{\partial t}\left(n+\Delta_{\rho} \psi\right)+C_{5}\left(n+\Delta_{\rho} \psi\right),
\end{aligned}
$$

we get from (6.11) and (6.12) that

$$
\begin{gathered}
-\frac{\partial}{\partial t}\left(e^{-c \psi}\left(n+\Delta_{\rho} \psi\right)\right)+\Delta^{\prime}\left(e^{-c \psi}\left(n+\Delta_{\rho} \psi\right)\right)+X\left(e^{-c \psi}\left(n+\Delta_{\rho} \psi\right)\right) \\
\geq e^{-c \psi}\left(-C_{6}-C_{7}\left(n+\Delta_{\rho} \psi\right)+C_{8} e^{\frac{1}{n-1} \psi}\left(n+\Delta_{\rho} \psi\right)^{1+\frac{1}{n-1}}\right) .
\end{gathered}
$$


Applying the Maximal Principle to function $e^{-c \psi}\left(n+\Delta_{\rho} \psi\right)$ in (6.13), we obtain

$$
n+\Delta_{\rho} \psi \leq C \exp \left\{c\left(\psi-\inf _{M} \psi\right)\right\}\left(1+\exp \left\{-\inf _{M} \psi\right\}\right) .
$$

Then (6.10) follows from (6.5).

Proposition 6.1. Assume that $M$ admits a Kähler-Ricci soliton $\left(g_{K S}, X\right)$. Then

(i) the metric $\omega_{g}+\frac{\sqrt{-1}}{2 \pi} \partial \bar{\partial} \bar{\varphi}$ is uniformly equivalent to the metric $\omega_{g}$;

(ii) we have a uniform estimate

$$
\|\bar{\varphi}\|_{C^{3}(M)} \leq C .
$$

Proof. It follows from (6.9) and Lemma 6.2 that

$$
0<c_{1} \leq n+\Delta_{\rho} \psi \leq c_{2} .
$$

It follows that

$$
c_{1} \leq n+\Delta_{\omega_{K S}}\left(\bar{\varphi}-\Psi_{0}\right) \leq c_{2} .
$$

Then (i) follows easily from this.

For the third derivative estimate, we consider Calabi's function

$$
S=g^{\prime i \bar{r}} g^{\prime s \bar{j}} g^{\prime k \bar{t}} \psi_{i \bar{j} k} \psi_{\bar{r} s \bar{t}}
$$

where $\left(g^{\prime} i \bar{j}\right)$ is the inverse of the Hermitian matrix function associated to the metric $\omega_{\rho}+\frac{\sqrt{-1}}{2 \pi} \partial \bar{\partial} \psi$. Note that $S$ is equal to the quantity

$$
\bar{g}^{i \bar{r}} \bar{g}^{s \bar{j}} \bar{g}^{k \bar{t}}\left(\bar{\varphi}-\Psi_{0}\right)_{i \bar{j} k}\left(\bar{\varphi}-\Psi_{0}\right)_{\bar{r} s \bar{t}},
$$

where $(\bar{g})^{i \bar{r}}$ denotes the inverse of the Hermitian matrix function associated to the metric $\omega_{\bar{\varphi}}$.

Following Calabi's computation as in [Ya], by Lemma 6.2, one can get

$$
\left(\Delta_{\rho}-\frac{\partial}{\partial t}\right)\left(S+c \Delta_{\rho} \psi\right) \geq C_{1} S-C_{2} .
$$

Here $c$ is sufficiently large, and $C_{1}$ and $C_{2}$ are uniform positive constants depending only on the metric $\omega_{K S}$. Then by the Maximal Principle, we see that $S \leq C$. Hence by part (i), we obtain

$$
\|\bar{\varphi}\|_{C^{3}(M)} \leq C^{\prime}
$$

Proof of Main Theorem. We shall prove that $\omega_{\bar{\varphi}_{t}}$ converges to a Kähler-Ricci soliton. First we show that the solution $\bar{\varphi}$ of (6.4) has uniformly bounded $C^{k}$-norms. Note that $\bar{\varphi}$ is a solution of the following equation:

$$
\frac{\partial \bar{\varphi}}{\partial t}=\log \frac{\operatorname{det}\left(g_{i \bar{j}}+\bar{\varphi}_{i \bar{j}}\right)}{\operatorname{det}\left(g_{i \bar{j}}\right)}+\operatorname{Re}(\bar{X}(\bar{\varphi}))+\bar{\varphi}-h+\operatorname{Re}\left(\theta_{\bar{X}}\right) .
$$

Differentiating this equation on $z_{k}$ in a coordinate chart of $M$ with local holomorphic coordinates $\left(z_{1}, \ldots, z_{n}\right)$, we have

$$
\left(\Delta^{\prime}-\frac{\partial}{\partial t}\right)\left(\frac{\partial \bar{\varphi}}{\partial z_{k}}\right)=g^{i \bar{j}} \frac{\partial g_{i \bar{j}}}{\partial z^{k}}-g^{\prime} \overline{i j} \frac{\partial g_{i \bar{j}}}{\partial z^{k}}-\bar{\varphi}_{k}-\left(h-\operatorname{Re}\left(\theta_{\bar{X}}-\bar{X}(\bar{\varphi})\right)\right)_{k} .
$$

Since $\Delta^{\prime}$ is uniformly elliptic and is at least of $C^{\alpha}(0<\alpha<1)$, by the standard Schauder estimates, we see that $\frac{\partial \bar{\varphi}}{\partial z_{k}}$ has a uniform $C^{2, \alpha}$-norm. Similarly, $\frac{\partial \bar{\varphi}}{\partial \bar{z}_{k}}$ has a uniform $C^{2, \alpha}$-norm. Hence, the $C^{3, \alpha}$-norm of $\bar{\varphi}$ is uniformly bounded. Repeating this process, one can easily show that the $C^{k}$-norm of $\bar{\varphi}$ is uniformly bounded for each integer $k \geq 0$. 
From the above arguments, we see that for any sequence of functions $\bar{\varphi}_{t}$, there is a subsequence which converges to a smooth function $\bar{\varphi}_{\infty}$ on $M$ in the $C^{k}$-topology. Next we show that $\omega_{g}+\frac{\sqrt{-1}}{2 \pi} \partial \bar{\partial} \bar{\varphi}_{\infty}$ is a Kähler-Ricci soliton. Let $\sigma_{t}=\exp \{t X\}$ and $\sigma^{\prime}=\sigma_{t}^{\prime}=\sigma_{t}^{-1} \cdot \bar{\rho}_{t}$. Since

$$
\int_{M}\left\|\bar{\partial}\left(\left(\sigma^{\prime}\right)^{*} \frac{\partial \varphi^{\prime}}{\partial t}\right)\right\|_{\omega_{\bar{\varphi}}}^{2} e^{\theta_{X}+X(\bar{\varphi})} \omega_{\bar{\varphi}}^{n}=\int_{M}\left\|\bar{\partial} \frac{\partial \varphi^{\prime}}{\partial t}\right\|_{\omega_{\varphi^{\prime}}}^{2} e^{\theta_{X}+X\left(\varphi^{\prime}\right)}\left(\omega_{\varphi^{\prime}}\right)^{n},
$$

then by (4.5), we have

$$
\int_{M}\left\|\bar{\partial}\left(\left(\sigma^{\prime}\right)^{*} \frac{\partial \varphi^{\prime}}{\partial t}\right)\right\|_{\omega_{\bar{\varphi}}}^{2} e^{\theta_{X}+X(\bar{\varphi})} \omega_{\bar{\varphi}}^{n}=-\frac{d \tilde{\mu}_{\omega_{g}}\left(\varphi^{\prime}\right)}{d t} .
$$

By the lower bound of $\tilde{\mu}_{\omega_{g}}\left(\varphi^{\prime}\right)$ [TZ2], one concludes that there is a sequence of $t_{i}$, $i=1,2, \ldots$, such that $\bar{X}_{i} \rightarrow X_{\infty}$ and

$$
\int_{M}\left\|\bar{\partial}\left(\left.\left(\sigma^{\prime}\right)^{*} \frac{\partial \varphi^{\prime}}{\partial t}\right|_{t_{i}}\right)\right\|_{\omega_{\bar{\varphi}_{t_{i}}}}^{2} \omega_{\bar{\varphi}_{t_{i}}}^{n} \rightarrow 0, \text { as } i \rightarrow \infty
$$

On the other hand, by (4.4), we have

$$
\frac{\sqrt{-1}}{2 \pi} \partial \bar{\partial}\left[\left(\sigma^{\prime}\right)^{*} \frac{\partial \varphi^{\prime}}{\partial t}\right]=-\operatorname{Ric}\left(\omega_{\bar{\varphi}}\right)+\omega_{\bar{\varphi}}+L_{X} \omega_{\bar{\varphi}} .
$$

Then the $C^{k}$-norm of $\left(\sigma^{\prime}\right)^{*} \frac{\partial}{\partial t} \varphi^{\prime}$ is uniformly bounded, so there exists a convergent subsequence of $\left.\left(\sigma^{\prime}\right)^{*} \frac{\partial}{\partial t} \varphi^{\prime}\right|_{t_{i}}$. Hence by (6.16) and Lemma 4.2, we conclude that $\left(\sigma^{\prime}\right)^{*} \frac{\partial}{\partial t} \varphi^{\prime}\left(t_{i}, \cdot\right)$ (still denoted by the same indices $t_{i}$ ) converges to zero in the $C^{k}$ topology, and consequently, by (6.17), Kähler metrics $\omega_{\bar{\varphi}_{t_{i}}}$ converge to a KählerRicci soliton associated to a holomorphic vector field $X_{\infty}$. By the uniqueness [TZ1] and [TZ2], we see that $X_{\infty}=X$ and the limit Kähler-Ricci soliton $\omega_{g}+\frac{\sqrt{-1}}{2 \pi} \partial \bar{\partial} \bar{\varphi}_{\infty}$ must be $\beta^{*} \omega_{K S}$ for some element $\beta \in \operatorname{Aut}_{r}(M)$. For simplicity, we may assume that $\beta$ is just the identity in $\operatorname{Aut}_{r}(M)$.

It remains to prove that $\omega_{\bar{\varphi}_{t}}$ converges to $\left(\omega_{g}+\frac{\sqrt{-1}}{2 \pi} \partial \bar{\partial} \bar{\varphi}_{\infty}, X\right)$ as $t$ goes to infinity. If this is false, then there is a convergent sequence $\omega_{\bar{\varphi}_{t_{i}}}$ whose limit is not $\left(\omega_{g}+\frac{\sqrt{-1}}{2 \pi} \partial \bar{\partial} \bar{\varphi}_{\infty}, X\right)$. But by (6.15) and the lower bound of $\tilde{\mu}_{\omega_{g}}\left(\varphi^{\prime}\right)$, we see that for any fixed number $\epsilon>0$ there is a sequence $s_{i} \in\left(-\epsilon+t_{i}, \epsilon+t_{i}\right), i=1,2, \ldots$, such that relation (6.16) holds for the sequence $\left.\left(\sigma^{\prime}\right)^{*} \frac{\partial \varphi^{\prime}}{\partial t}\right|_{t=s_{i}}$. It follows from (6.17) that $\omega_{\bar{\varphi}_{s_{i}}}$ converges to $\left(\omega_{g}+\frac{\sqrt{-1}}{2 \pi} \partial \bar{\partial} \bar{\varphi}_{\infty}, X\right)$ in the $C^{k}$ sense. On other hand, on each interval $\left[-\epsilon+t_{i}, \epsilon+t_{i}\right], \bar{\varphi}-\bar{\varphi}_{s_{i}}$ satisfies the following Monge-Ampère flow:

$$
\frac{\partial\left(\bar{\varphi}-\bar{\varphi}_{s_{i}}\right)}{\partial t}=\log \frac{\left(\omega_{\bar{\varphi}_{s_{i}}}+\frac{\sqrt{-1}}{2 \pi} \partial \bar{\partial}\left(\bar{\varphi}-\bar{\varphi}_{s_{i}}\right)\right)^{n}}{\omega_{\bar{\varphi}_{s_{i}}}^{n}}+\operatorname{Re}\left(\bar{X}\left(\bar{\varphi}-\bar{\varphi}_{s_{i}}\right)\right)+\left(\bar{\varphi}-\bar{\varphi}_{s_{i}}\right)+G,
$$

where

$$
H=-\left.\frac{\partial \bar{\varphi}}{\partial t}\right|_{s_{i}}+\operatorname{Re}\left(\theta_{\bar{X}}-\theta_{\bar{X}_{s_{i}}}\right)+\operatorname{Re}\left(\left(\bar{X}-\bar{X}_{s_{i}}\right) \bar{\varphi}_{s_{i}}\right) .
$$

Note that $\|H\|_{C^{k}(M)} \leq C \epsilon$, when $i$ is sufficiently large. Thus by using the implicit function theorem, we see that

$$
\left\|\bar{\varphi}_{t}-\bar{\varphi}_{s_{i}}\right\|_{C^{k}(M)} \leq \delta(\epsilon), \forall t \in\left[-\epsilon+t_{i}, \epsilon+t_{i}\right],
$$

when $i$ is sufficiently large. Here $\delta(\epsilon) \rightarrow 0$ as $\epsilon \rightarrow 0$. Since $\epsilon$ can be chosen to be small, the sequence $\bar{\varphi}_{t_{i}}$ will converge to $\bar{\varphi}_{\infty}$, which contradicts the assumption. The proof is completed. 
Remark 6.1. To get the $C^{2}$-estimate and $C^{3}$-estimate of $\bar{\varphi}_{t}$, we used (6.9) instead of (6.14). The reason for this is that in (6.9), the holomorphic vector field $X$ is fixed and $X\left(\psi_{t}\right)$ is uniformly bounded according to a result in [Z $\mathbf{Z h}$, while in (6.14), we do not know how to bound $\operatorname{Re}\left(\bar{X}_{t}\left(\bar{\varphi}_{t}\right)\right)$.

Remark 6.2. Following arguments in Section 10 in [CT1, one can further prove that the evolved metrics $\omega_{\bar{\varphi}_{t}}$ of (6.17) will exponentially converge to a Kähler-Ricci soliton with respect to $X$ in the sense of Cheeger-Gromov. More precisely, there is a family of automorphisms $\beta_{t} \in \operatorname{Aut}_{r}(M)$ with $\left\|\beta_{t}-I d\right\| \leq C$ such that for any integer $k \geq 0$ it holds that

$$
\left\|D^{k}\left[\beta_{t}^{*}\left(\operatorname{Ric}\left(\omega_{\bar{\varphi}_{t}}\right)-\omega_{\bar{\varphi}_{t}}-L_{X} \omega_{\bar{\varphi}_{t}}\right)\right]\right\|_{g_{K S}} \leq C_{k}(\epsilon) e^{-2\left(\lambda_{2}-1-\epsilon\right) t} \rightarrow 0 \text {, as } t \rightarrow \infty,
$$

where $D^{k}$ denotes the covariant derivative of degree $k$ with respcet to the KählerRicci soliton $g_{K S}$ and $\epsilon>0$ is a small number and $\lambda_{2}>1$ is the second nonzero eigenvalue of operator $\triangle_{g_{K S}}+X(\cdot)$ on the $K_{X}$-invariant functions space.

\section{APPENDIX}

In this appendix, we outline a proof of Lemma 1.1 which is taken from [ST]. The proof consists of three main steps.

Step 1. Let $u=-h_{t}$. Then we show that $u$ is uniformly bounded from below. To prove this, we need to introduce Perelman's $W$-functional for a pair $(g, f)$ :

$$
W(g, f)=\int_{M}\left[\left(R(g)+|D f|^{2}\right)+f\right] e^{-f} d V_{g},
$$

where $g$ is a Riemanian metric on $M$ and $R(g)$ denotes the scalar curvature of $g$ and where $f$ is a smooth function on $M$ which satisfies a normalization condition

$$
\int_{M} e^{-f} d V_{g}=\int_{M} d V_{g} \equiv V
$$

Let

$$
\mu(g)=\inf \{W(g, f) \mid f \text { satisfies }(A .2)\} .
$$

It is easy to see that $\mu(g)$ can be attained by some $f$. In [P1], it was shown that $\mu(g(t))$ is increasing if $g(t)$ is a family of evolved Riemanian metrics along the Ricci flow. Since in our case of Kähler-Ricci flow (0.1),

$$
R(g(t))=4(-\triangle u+n),
$$

we have

$$
\begin{aligned}
C & \leq \mu(g(t)) \leq W\left(g(t), h_{t}\right) \\
& =\int_{M}\left[4\left(-\triangle u+|\nabla u|^{2}+n\right)+u\right] e^{-u} \omega_{\varphi}^{n}=4 n \int_{M} \omega_{g_{0}}^{n}+\int_{M} u e^{-u} \omega_{\varphi}^{n},
\end{aligned}
$$

where $\triangle$ and $\nabla$ denote the $\bar{\partial}$-Lapalace operator and the covariant derivative of type $(1,0)$ associated to metric $g(t)$, respectively. Thus

$$
\int_{M} u e^{-u} \omega_{\varphi}^{n} \geq-C_{1}
$$

By (1.3), we have

$$
\frac{d u}{d t}=\Delta u+u+a
$$


for some constants $a=a_{t}$. Using (A.2), we get

$$
a=-\frac{1}{V} \int_{M} u e^{-u} \omega_{\varphi}^{n} \leq C_{1} .
$$

On the other hand, by using the Maximal Principle, it is known that scalar curvature $R=R(g(t))$ is uniformly bounded from below. Thus

$$
\frac{\partial u}{\partial t}=-R+n+u+a \leq C_{2}+u
$$

where $R=-\Delta u+n$ is the scalar curvature of $g(t)$ after a scale for the case of Kähler metrics.

Now we assume that $u\left(t_{0}\right)\left(x_{0}\right)<-2 C_{2}<0$ for some time $t_{0}$ and some $x_{0} \in M$. So it holds that

$$
\left.u\left(t_{0}\right)\right|_{U} \leq-2 C_{2}
$$

for some neighborhood $U$ of $x_{0}$. Thus

$$
\left.\frac{\partial u}{\partial t}\left(t_{0}\right)\right|_{U} \leq C_{2}+u<-C_{2}<0 \text {. }
$$

It follows that

$$
\left.u(t)\right|_{U} \leq-C_{2} e^{t-t_{0}} \leq-C_{3} e^{t}
$$

for any $t \geq t_{0}$.

We choose some constants $b_{t}$ so that $\tilde{\varphi}=\tilde{\varphi}_{t}=\varphi_{t}+b_{t}$ and $u=\frac{\partial \tilde{\varphi}}{d t}$. Then by (A.6), we have

$$
\left.\tilde{\varphi}(t)\right|_{U} \leq-C_{3}^{\prime} e^{t}
$$

for any $t \geq t_{0}$. Since $\tilde{\varphi}(t)$ is an almost subharmonic function, one can apply the Green's formula to obtain

$$
\max _{M} \tilde{\varphi}_{t} \leq \max _{U} \tilde{\varphi}_{t}+C\left(g_{0}\right) \leq-C_{3}^{\prime} e^{t}+C\left(g_{0}\right),
$$

for any $t \geq t_{0}$. On the other hand, by (A.5), one sees

$$
\frac{\partial}{\partial t}(u-\tilde{\varphi}) \leq C_{2}
$$

It follows that

$$
\max _{M} \tilde{\varphi}_{t} \geq-C_{2} t-\max _{M}\left(u_{0}-\tilde{\varphi}_{0}\right) .
$$

(A.8) is a contradiction to (A.7) for sufficiently large $t$. This implies that $u(t) \geq$ $-2 C_{2}$ for any $t$ and so Step 1 is proved.

Step 2. Choose $B$ so that $u+B>0$. Then we claim

$$
|\nabla u|^{2} \leq C(u+B)
$$

and

$$
-\triangle u \leq C(u+B) .
$$

(A.9) and (A.10) can be proved by using the standard Maximal Principle for the parabolic operator $\frac{\partial}{\partial t}-\triangle$ (see [ST] for details). For simplicity, we outline a proof for $($ A.9).

By (A.4), we have

$$
\left(\frac{\partial}{\partial t}-\triangle\right)(\triangle u)=-|\nabla \bar{\nabla} u|^{2}+\triangle u
$$


and

$$
\left(\frac{\partial}{\partial t}-\triangle\right)|\nabla u|^{2}=-|\nabla \nabla u|^{2}-|\nabla \bar{\nabla} u|^{2}+|\nabla u|^{2} .
$$

Let $H=\frac{|\nabla u|^{2}}{u+B}$. Then

$$
\begin{aligned}
\left(\frac{\partial}{\partial t}-\triangle\right) H & =\frac{|\nabla u|^{2}(B-a)}{(u+B)^{2}}-\frac{|\nabla \nabla u|^{2}+|\nabla \bar{\nabla} u|^{2}}{u+B} \\
& +2 \frac{\left\langle\nabla u, \nabla|\nabla u|^{2}\right\rangle}{(u+B)^{2}}-2 \frac{|\nabla u|^{4}}{(u+B)^{3}} .
\end{aligned}
$$

After a simple estimate of the last three terms on the right side of (A.11), one shows

$$
\left(\frac{\partial}{\partial t}-\triangle\right) H \leq \frac{|\nabla u|^{2}(B-a)}{(u+B)^{2}}+(2-\epsilon) \frac{\langle\nabla u, \nabla H\rangle}{u+B}-\frac{\epsilon}{2} \frac{|\nabla u|^{4}}{(u+B)^{3}},
$$

for some small $\epsilon$. Thus one can use the Maximal Principle to get

$$
\max _{M} H \leq C .
$$

This proves (A.9).

By (A.9), we get

$$
u(x, t) \leq C \operatorname{Diam}(M, g(t))^{2}+C^{\prime} .
$$

So to complete Lemma 1.1, it suffices to prove that the diameters $\operatorname{Diam}(M, g(t))$ of $M$ are uniformly bounded. Note that we can also get from (A.10) and (A.12) that

$$
R(g(t)) \leq C \operatorname{Diam}(M, g(t))^{2}+C^{\prime}
$$

and

$$
|\nabla u| \leq C \operatorname{Diam}(M, g(t))+C^{\prime} .
$$

Step 3. Prove

$$
\operatorname{Diam}(M, g(t)) \leq C
$$

We use an argument by contradiction to prove (A.15). The proof is similar to one of Perelman's noncollapsing theorems ([P1] $)$. Let $x=x_{t} \in M$ be a minimal point of function $u(t, y)$ and denote $d_{t}(z)=\operatorname{dist}_{t}(x, z)$. Let $B_{t}\left(k_{1}, k_{2}\right)=\left\{z \mid 2^{k_{1}} \leq d_{t}(z) \leq\right.$ $\left.2^{k_{2}}\right\}$ be an annuli in $M$. Then by using the noncollapsing result of Ricci flow ([P1], $[\mathrm{KL}$ ) together with (A.13) and (A.14), one sees that the following statement is true ([ST]): If $\operatorname{Diam}(M, g(t))$ is large enough, then for every $\epsilon>0$ there exists $B_{t}\left(k_{1}, k_{2}\right)$ such that

$$
\operatorname{Vol}\left(B_{t}\left(k_{1}, k_{2}\right)\right)<\epsilon \text { and } \operatorname{Vol}\left(B_{t}\left(k_{1}, k_{2}\right)\right) \leq 2^{10 n} \operatorname{Vol}\left(B_{t}\left(k_{1}+2, k_{2}-2\right)\right) .
$$

Moreover, there exists $r_{1}$ and $r_{2}$ and a uniform constant $C$ such that $2^{k_{1}} \leq r_{1} \leq$ $2^{k_{1}+1}, 2^{k_{2}} \leq r_{2} \leq 2^{k_{2}+1}$ and

$$
\int_{B_{t}\left(r_{1}, r_{2}\right)} R(g(t)) \omega_{\varphi_{t}}^{n} \leq C \operatorname{Vol}\left(B_{t}\left(k_{1}, k_{2}\right)\right) .
$$

Now we suppose that there is a sequence $t_{i}$ such that $\operatorname{Diam}\left(M, g\left(t_{i}\right)\right) \rightarrow \infty$. Then by the above statement there are sequences $\left\{k_{1}^{i}\right\},\left\{k_{2}^{i}\right\},\left\{r_{1}^{i}\right\}$ and $\left\{r_{2}^{i}\right\}$ such that (A.16) and (A.17) are satisfied for these sequences. Let $f_{i}$ be a sequence of smooth cut-off functions on $\mathbb{R}$ such that $f_{i} \equiv 1$ on $\left[2^{k_{1}^{i}+2}, 2^{k_{2}^{i}-2}\right]$ and $f_{i} \equiv 0$ on 
$\left(-\infty, r_{1}^{i}\right] \cup\left[r_{2}^{i}, \infty\right)$. Let $u_{i}(z)=e^{C_{i}} f_{i}\left(d_{t}(z)\right)$ be a sequence of functions on $M$ such that $\int_{M} u_{i}^{2} \omega_{\varphi_{t_{i}}}^{n}=\int_{M} \omega_{g_{0}}^{n}$ by choosing suitable constants $C_{i}$. Since

$$
\operatorname{Vol}\left(B_{t_{i}}\left(k_{1}^{i}, k_{2}^{i}\right)\right)<\epsilon_{i} \rightarrow 0,
$$

it is easy to see that $C_{i} \rightarrow 0$.

By the monotonicity of $\mu(g(t))$, we have

$$
\begin{aligned}
C & \leq \mu\left(g\left(t_{i}\right)\right) \leq W\left(g\left(t_{i}\right),-2 \ln u_{i}\right) \\
& =e^{2 C_{i}} \int_{B_{t_{i}}\left(r_{i}^{1}, r_{i}^{2}\right)}\left(4\left|f_{i}^{\prime}\left(\operatorname{dist}_{t_{i}}(z)\right)\right|^{2}-2 f_{i}^{2} \ln f_{i}\right) \omega_{\varphi_{t_{i}}}^{n} \\
& +\int_{B_{t_{i}}\left(r_{i}^{1}, r_{i}^{2}\right)} R\left(g\left(t_{i}\right)\right) u_{i}^{2} \omega_{\varphi_{t_{i}}}^{n}-2 C_{i} .
\end{aligned}
$$

On the other hand, by (A.16) and (A.17), we estimate

$$
\begin{aligned}
\int_{B_{t_{i}}\left(r_{1}^{i}, r_{2}^{i}\right)} R\left(g\left(t_{i}\right)\right) u_{2}^{i} \omega_{\varphi_{t_{i}}}^{n} & \leq e^{2 C_{i}} \int_{B_{t_{i}}\left(r_{1}^{i}, r_{2}^{i}\right)} R\left(g\left(t_{i}\right)\right) \omega_{\varphi_{t_{i}}}^{n} \\
& \leq C_{1} e^{2 C_{i}} \operatorname{Vol}\left(B_{t_{i}}\left(k_{1}^{i}, k_{2}^{i}\right)\right) \\
& \leq C_{1} e^{2 C_{i}} 2^{10 n} \operatorname{Vol}\left(B_{t_{i}}\left(k_{1}^{i}+2, k_{2}^{i}-2\right)\right) \\
& \leq C_{1} 2^{10 n} \int_{M} u_{i}^{2} \omega_{\varphi_{t_{i}}}^{n}=C_{1} 2^{10 n} \int_{M} \omega_{g_{0}}^{n} .
\end{aligned}
$$

Similarly by (A.16), we have

$$
e^{2 C_{i}} \int_{B_{t_{i}}\left(r_{i}^{1}, r_{i}^{2}\right)}\left(4\left|f_{i}^{\prime}\left(\operatorname{dist}_{t_{i}}(z)\right)\right|^{2}-2 f_{i}^{2} \ln f_{i}\right) \omega_{\varphi_{t_{i}}}^{n} \leq C_{2} 2^{10 n} \int_{M} \omega_{g_{0}}^{n}
$$

Thus inserting (A.19) and (A.20) into (A.18), we get

$$
C \leq C_{3}-2 C_{i} \rightarrow-\infty,
$$

which is impossible. Therefore, (A.15) is true.

\section{REFERENCES}

[AT] Alexander, H.J. and Taylor, B.A., Comparison of two capacities in $\mathbb{C}^{n}$, Math. Z., 186 (1984), 407-417. MR0744831 (85k:32034)

[BT] Bedford, E. and Taylor, B.A., A new capacity for plursubharmonic functions, Acta Math., 149 (1982), 1-40. MR0674165 (84d:32024)

[Ca] Cao, H.D., Deformation of Kähler metrics to Kähler-Einstein metrics on compact Kähler manifolds, Invent. Math., 81 (1985), 359-372. MR.0799272 (87d:58051)

[CT1] Chen, X.X. and Tian, G., Ricci flow on Kähler-Einstein surfaces, Invent. Math., 147 (2002), 487-544. MR.1893004 (2003c:53095)

[CT2] Chen, X.X. and Tian, G., Ricci flow on Kähler-Einstein manifolds, Duke Math. J., 131 (2006), 17-73. MR.2219236

[CTZ] Cao, H.D., Tian, G., and Zhu, X.H., Kähler-Ricci solitons on compact Kähler manifolds with $c_{1}(M)>0$, Geom and Funct. Anal., 15 (2005), 697-719. MR2221147

[F1] Futaki, A., An obstruction to the existence of Kähler-Einstein metrics, Invent. Math., 73 (1983), 437-443. MR0718940 (84j:53072)

[F2] Futaki, A., Kähler-Einstein metrics and integral invariants, Lecture Notes in Math, 1314 (1988), Springer-Verlag, Berlin, New-York. MR0947341 (90a:53053)

[Ha] Hamilton, R.S., Three manifolds with positive Ricci Curvature, J. Diff. Geom., 17 (1982), 255-306. MR0664497 (84a:53050)

[KL] Kleiner. B. and Lott, J., Notes on Perelman's papers, preprint, 2003.

[Ko] Kolodziej, S., The complex Monge-Ampère equation, Acta Math., 180 (1998), 69-117. MR1618325 (99h:32017) 
[Ma] Mabuchi, T., K-energy maps integrating Futaki invariants, Tohöku Math. J., 38 (1986), 245-257. MR0867064 (88b:53060)

[P1] Perelman, G., The entropy formula for the Ricci flow and its geometric applications, preprint, 2002.

[P2] Perelman, G., unpublished.

[ST] Sesum, N. and Tian, G., Perelman's argument for uniform bounded scalar curvature and diameter along the Kähler-Ricci flow, preprint, 2005.

[Ti] Tian, G., Kähler-Einstein metrics with positive scalar curvature, Invent. Math., 130 (1997), 1-37. MR1471884 (99e:53065)

[TZ1] Tian, G. and Zhu, X.H., Uniqueness of Kähler-Ricci solitons, Acta Math., 184 (2000), 271-305. MR:1768112 (2001h:32040)

[TZ2] Tian, G. and Zhu, X.H., A new holomorphic invariant and uniqueness of Kähler-Ricci solitons, Comm. Math. Helv., 77 (2002), 297-325. MR1915043(2003i:32042)

[Ya] Yau, S.T., On the Ricci curvature of a compact Kähler manifold and the complex Monge-Ampère equation, I, Comm. Pure Appl. Math., 31 (1978), 339-411. MR0480350 (81d:53045)

[Zh] Zhu, X.H., Kähler-Ricci soliton type equations on compact complex manifolds with $C_{1}(M)>0$, J. Geom. Anal., 10 (2000), 759-774. MR1817785(2002c:32042)

Department of Mathematics, Princeton University, Princeton, New Jersey 08544

E-mail address: tian@math.princeton.edu

Department of Mathematics, Peking University, Beijing, 100871, People's Republic OF CHINA

E-mail address: xhzhu@math.pku.edu.cn 